\title{
Interference-Aware NOMA for Cellular-Connected UAVs: Stochastic Geometry Analysis
}

\author{
Wee Kiat New, Student Member, IEEE, Chee Yen Leow, Member, IEEE, \\ Keivan Navaie, Senior Member, IEEE, Yanshi Sun, Student Member, IEEE, and Zhiguo Ding, Fellow, IEEE
}

\begin{abstract}
Efficiency of cellular-connected UAVs is challenged by spectrum inefficiency, limited number of concurrent connectivity, and strong interference. To overcome these issues, in this paper, we study the performance of downlink non-orthogonal multiple access for cellular-connected UAVs. We develop a novel framework based on stochastic geometry for the co-existence of aerial users (AUs) and terrestrial users (TUs), where the spatial distribution of the base stations (BSs) follows a Poisson Point Process. In our analysis, two user association policies and two types of receive antennas are considered while an intercell interference coordination (ICIC) technique is also in place. As the main performance measures, we then analytically derive the coverage probability and average rate of AUs and TUs. These derivations are then used to provide quantitative insights on the impact of different system parameters and settings including AU's altitude, TU's distance from the BS, power allocation, successive interference cancellation (SIC) constraints, user association policy, antenna beamwidth, and the number of coordinated BSs. Based on our analysis we then propose an interference-aware scheme based on maximum-SINR user association, directional antenna, and ICIC. A benchmark scheme based on minimum-distance user association, omni-directional antenna, and without ICIC is considered. Compared to the benchmark scheme, our proposed scheme improves the AU's coverage probability by threefold and TU's average rate by sixfold. Compared to the orthogonal multiple access, our proposed scheme trades off a slight reduction in the AU's coverage probability $(\sim 1 \%)$ with a significant increase in the achieved rate of the TUs (603Kbps/resource block).
\end{abstract}

Index Terms-Non-Orthogonal Multiple Access, CellularConnected UAVs, Stochastic Geometry, Aerial Users, Terrestrial Users

\section{INTRODUCTION}

In recent years, unmanned aerial vehicles (UAVs) have gained significant attention among the wireless communication research community. On one hand, UAVs have been

Manuscript received: October 13, 2020; revised February 4, 2021; accepted April 12, 2021. Part of this paper was presented in IEEE GLOBECOM 2020 [1]. This work was supported in part by the Ministry of Higher Education Malaysia and Universiti Teknologi Malaysia under grant numbers FRGS/1/2020/TK0/UTM/02/68, 08G83, 09G15, 4J416 and 04G37. (Corresponding author: Chee Yen Leow.)

Wee Kiat New and Chee Yen Leow are with the Wireless Communication Centre, School of Electrical Engineering, Faculty of Engineering, Universiti Teknologi Malaysia, 81310 Skudai, Johor, Malaysia. (email: aven.wknew@yahoo.com; bruceleow@utm.my).

Keivan Navaie is with the School of Computing and Communications, Lancaster University, Lancaster, United Kingdom LA1 4WA. (e-mail: k.navaie@lancaster.ac.uk).

Yanshi Sun is with the School of Information Science and Technology, University of Science and Technology of China, Hefei, China. (e-mail: sys@mail.ustc.edu.cn).

Zhiguo Ding is with the School of Electrical and Electronic Engineering, the University of Manchester, UK. (e-mail: zhiguo.ding@manchester.ac.uk). studied for aerial communication platforms, which employ UAVs as aerial base stations (BSs) or aerial relays. On the other hand, UAVs have been treated as aerial users (AUs) for civil applications such as agriculture, construction, delivery, and surveillance. In this paper, we focus on the latter.

The idea of connecting AUs to the existing cellular network has been proposed. Such an idea is known as cellularconnected UAVs. Unlike point-to-point links over unlicensed spectrum, cellular-connected UAVs have the potential of enabling AUs to operate beyond visual line-of-sight (LOS). Nevertheless, existing cellular networks are primarily designed to serve terrestrial users (TU)s only. For example, terrestrial BSs usually tilt their antennas downward to enhance the desired signal and reduce the inter-cell interference (ICI) of TUs. This causes AUs to be served by the BS's sidelobe and therefore might suffer significant performance degradation [2].

Furthermore, ground-to-air channels exhibit a rather different behavior than that of the ground-to-ground channels [3]. In particular, AUs that are hovering at a high altitude experience a higher probability of LOS to BSs as compared to TUs. Consequently, AUs experience stronger communication links which leads two contrasting effects: i) stronger link to its associated BS, and ii) stronger ICI from neighboring BSs. Recent measurement campaigns and simulation studies have further shown that the latter brings more adversities than benefits [4]-[7].

Extensive research efforts have therefore been made to provide reliable connectivity to new AUs to ensure their safe operation. For example, [8], [9] have suggested to decrease AUs' altitude to alleviate the ICI effect and [10], [11] have further suggested to exploit the coverage extension feature to ensure a reliable cell-acquisition and ubiquitous mobility for the AUs. New techniques have also been developed to handle the high ICI at the AUs. For instance, [12] has proposed a cooperative beamforming and interference cancellation scheme and [13] has examined the use of tilted directional antenna. Nevertheless, most of these works only focus on orthogonal multiple access (OMA).

Although OMA ensures less co-channel interference, its spectral efficiency and number of concurrent connectivity are limited. This is because, in OMA, each orthogonal resource block (RB) can only be assigned to a single user. Hence, to improve the spectrum efficiency and number of concurrent connectivity, one may employ non-orthogonal multiple access (NOMA). NOMA is a multiple access that enables multiple users to share the same orthogonal RB by leveraging superposition coding at the transmitter and successive interference 
cancellation (SIC) at the receiver. Its superiority over OMA has also been proven in several studies [14], [15].

Furthermore, some works have considered the applications of NOMA for cellular-connected UAVs [16]-[20]. Nevertheless, these works only focus on uplink communication. To the best of our knowledge, only few works consider downlink NOMA for co-existence of AUs and TUs. For instance, [21] has proposed a robust NOMA scheme for AU and TU to serve command and control (C\&C) and data links, respectively, [22] has derived the outage probability for co-existence of $\mathrm{AU}$ and TU in a single cell network, and [23] has proposed an interference cancellation scheme where the ICI is generated by a BS only. The impact of ICI and various network parameters have not been studied and the performance of NOMA for AUs and TUs remains unclear. Nevertheless, as it was shown in [24], NOMA might be a perfect fit to serve AUs and TUs for $\mathrm{C} \& \mathrm{C}$ and data links, respectively, due to their downlink asymmetricity.

Due to the aforementioned motivations, we study the performance of downlink NOMA for co-existing AUs and TUs using the tools of stochastic geometry. Stochastic geometry is a mathematical framework that studies the average behaviors over random spatial patterns [25]. Stochastic geometry has increasingly been used as an important analytical tool for evaluating the impact of different network parameters and benchmarking, while considering the spatial distributions of BSs and users [26]. One common approach is to model the BSs in cellular networks as a realization of a random spatial point process and then characterize the system performance such as signal-to-interference-plus-noise ratio (SINR) and/or rates. Such a methodology can often reveal key dependencies in the systems and provide useful guidance on the system designs and settings. For a more rigorous introduction on stochastic geometry for wireless networks, we refer the interested readers to [25]-[29].

Stochastic geometric frameworks for uplink and downlink NOMA have been investigated in the related literature, e.g., [30]-[34]. Nevertheless, the existing frameworks cannot be directly applied to evaluate the performance of NOMA for co-existing AUs and TUs in cellular networks. Specifically, it is essential to consider a three dimensional (3D) spatial space and unique characteristics of aerial propagation such as mainlobe/sidelobe effects, probability of LOS/NLOS, and $3 \mathrm{D}$ receiving coverage. Related works such as [19], [20] only consider a single-cell uplink NOMA. To the best of our knowledge, there is no analytical framework available in the literature to evaluate the performance of downlink NOMA for co-existence of AUs and TUs in cellular networks for different types of user association policies, receive antenna deployments, and number of ICI coordination (ICIC) in a 3D spatial setting.

In this paper, we propose a novel stochastic geometric framework to study the performance of downlink NOMA for co-existence of AUs and TUs in cellular networks, while incorporating two types of user association policies, two types of receive antennas, and ICIC. This proposed framework facilitates the performance analysis for the impact of various network parameters as well as the ICI effect on both AUs and TUs. In addition, we propose a novel interference-aware scheme that can efficiently serve AUs, and TUs for C\&C, and data links, respectively. Note that, in downlink, AUs requires a low rate but highly reliable connectivity (e.g., high coverage probability) and TUs requires massive data rate (e.g., high data rate). Therefore, a high AU's coverage probability and a high TU's average rate are desired.

The main contributions of this paper are summarized as follows:

- We propose a novel stochastic geometric framework, where terrestrial BSs are spatially distributed according to a homogeneous Poisson point process (PPP) and each $\mathrm{AU}$ is paired with a TU using NOMA. Furthermore, we incorporate minimum-distance and maximum-SINR based user associations, directional and omni-directional antennas, and also ICIC into the proposed framework.

- We further derive computationally tractable expressions for the coverage probabilities and average rates of the AUs and TUs, through a set of analytical derivations and calculations presented and proved in a series of lemmas and theorems.

- Using the derived analytical expressions, we further analyze the impact of various network settings and parameters. Our discussions further provide quantitative and intuitive insights on the system characteristics at a fundamental level, as well as practical guidelines for efficient system design.

- To efficiently serve AUs and TUs, we propose an interference-aware scheme that combines the use of maximum-SINR based user association, directional antenna with fixed beamwidth, and ICIC. Analytical and simulation results are presented to verify the superiority of the proposed scheme as compared to other schemes based on different combinations.

We note that this paper is a substantial extension of our previous work [1] by considering a new set of features and effects including noise, SIC constraints, maximum-SINR based user association, directional antenna with fixed beamwidth, and ICIC. In particular, in this paper, the expressions of the coverage probability of AUs and TUs are much more generalized than those of our previous work [1]. Furthermore, in this paper, we derive the average rate of AUs and TUs. This enables us to present the rate region of AUs and TUs. In addition, this paper provides extensive simulation results for various network parameters and settings.

The rest of the paper is organized as follows: Section II details the system model and the proposed framework. Section III presents the performance analysis. The simulation and analytical results are then provided in Section IV, followed by the conclusions drawn in Section V.

Notations: In this paper, scalar variables are denoted by italic letters (e.g., c) and vectors are denoted by boldface italic letters (e.g., c). Besides, $(\cdot)^{T}$ denotes transpose and $\|\cdot\|$ denotes Euclidean norm. $\mathcal{C}(x, c)$ denotes a circle centered at point $x$ with a radius of $c, \min / \max (\cdot, \cdot)$ denotes the minimum/maximum value of the argument, and $\log (\cdot)$ denotes logarithm with base 2 . In addition, $\mathbb{E}[\cdot]$ denotes the expectation, $f_{x}(\cdot)$ denotes the probability density function of $x$, and 


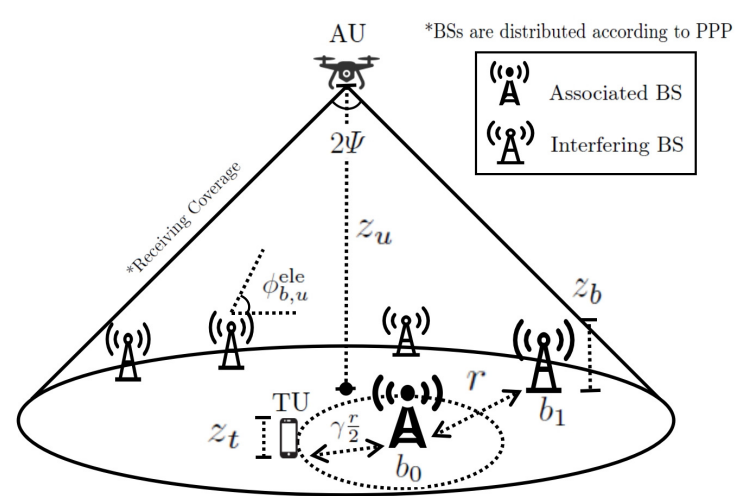

Figure 1: Illustration of the proposed framework: the AU is equipped with a directional antenna.

$F_{x}(\cdot)$ denotes the cumulative distribution function of $x$. The notation $\mathbf{1}_{c}\{\cdot\}$ is an indication function for condition $c$ and $[\cdot]_{c}^{+/-}$denotes the argument is lower/upper bounded by $c$.

\section{System Model And The Proposed Framework}

\section{A. Poisson Point Process}

In this framework, we consider a downlink wireless communication system with multiple terrestrial BSs. We assume the BSs are distributed according to a homogeneous PPP $\Phi_{\mathcal{B}}$ with intensity $\lambda$ and at a fixed height of $z_{b}$. We use subscript $b$ to denote the BSs or points of the point process, i.e., $b \in \Phi_{\mathcal{B}}$. We further consider a collection of mobile AUs, where their locations follow an independent stationary point process and each AU is assumed to be paired with a TU over an orthogonal $\mathrm{RB}$ of the same associated BS via NOMA. Without loss of generality, we focus on the performance of a typical pair of $\mathrm{AU}$ and TU, as shown in Fig. 1. We denote $b_{0} \in \Phi_{\mathcal{B}}$ as the associated $\mathrm{BS}$, and $r$ as the distance from $\mathrm{BS} b_{0}$ to its nearest BS $b_{1}$, where $b_{1} \in \Phi_{\mathcal{B}} /\left\{b_{0}\right\}$. By Slivynak's theorem, the typical $\mathrm{AU}$ is assumed to be located at the origin with a fixed altitude of $z_{u}$. In addition, we assume TU is uniformly distributed at the circle $\mathcal{C}\left(b_{0}, \gamma \frac{r}{2}\right)$ at a fixed height of $z_{t}$, where $\gamma \in(0,1]$ is a network parameter that determines the size of the circle and $z_{u}>z_{b}>z_{t}$.

We denote $\boldsymbol{w}_{b}^{\mathbf{B S}}=\left[x_{b}, y_{b}, z_{b}\right]^{T}, \boldsymbol{w}^{\mathrm{TU}}=\left[x_{t}, y_{t}, z_{t}\right]^{T}$, and $\boldsymbol{w}^{\mathrm{AU}}=\left[0,0, z_{u}\right]^{T}$ as the 3D location of the BS $b$, TU, and AU, respectively. The Euclidean distance between TU and BS $b$ is:

$$
d_{b, t}=\left\|\boldsymbol{w}_{b}^{\mathrm{BS}}-\boldsymbol{w}^{\mathrm{TU}}\right\|
$$

and the Euclidean distance between $\mathrm{AU}$ and BS $b$ is:

$$
d_{b, u}=\left\|\boldsymbol{w}_{b}^{\mathrm{BS}}-\boldsymbol{w}^{\mathrm{AU}}\right\| .
$$

Let $\hat{\boldsymbol{w}}_{b}^{\mathrm{BS}} \triangleq\left[x_{b}, y_{b}\right]^{T}, \hat{\boldsymbol{w}}^{\mathrm{TU}} \triangleq\left[x_{t}, y_{t}\right]^{T}, \hat{\boldsymbol{w}}^{\mathrm{AU}} \triangleq[0,0]^{T}$ be the 2D location of the $\mathrm{BS} b$, TU, and AU, respectively. The horizontal distance between $\mathrm{BS} b$ and AU, and BS $b$ and $\mathrm{TU}$ are $r_{b, u}=\left\|\hat{\boldsymbol{w}}_{b}^{\mathrm{BS}}-\hat{\boldsymbol{w}}^{\mathrm{AU}}\right\|$, and $r_{b, t}=\left\|\hat{\boldsymbol{w}}_{b}^{\mathrm{BS}}-\hat{\boldsymbol{w}}^{\mathrm{TU}}\right\|$, respectively. For brevity, we further denote $r_{u}=r_{b_{0}, u}$ and $r_{t}=r_{b_{0}, t}$. The vertical distance between BS $b$ and AU, and BS $b$ and TU are $\triangle z_{u}=\left|z_{u}-z_{b}\right|$, and $\triangle z_{t}=\left|z_{t}-z_{b}\right|$, respectively.

\section{B. Antenna Modeling}

At the user equipment, we consider two types of receive antennas: directional antenna with fixed beamwidth, and omni-directional antenna. For the former, we assume AU is equipped with a directional antenna, where the antenna is pointing directly below the $\mathrm{AU}$, i.e., $\hat{\boldsymbol{w}}^{\mathrm{AU}}$. The azimuth and elevation half-power beamwidth of the directional antenna are assumed equal and they are characterized by $2 \Psi$, where $\Psi \in\left(0, \frac{\pi}{2}\right)$. According to [3], [13], [35], [36], the receive antenna gain is $G_{u}^{\mathrm{rx}}(\Psi)=\frac{2.2856}{\Psi^{2}}$ within the receiving coverage and approximately zero outside of the receiving coverage. The receiving coverage of the AU is a cone, where the width of the receiving coverage at the height of $z$ is $r_{c}(z, \Psi)=\left\|z_{u}-z\right\| \tan \Psi$ and $z_{u} \geq z$. The set of BSs within the receiving coverage of the $\mathrm{AU}$ is denoted by

$$
\Phi_{\mathcal{C}}^{u}=\left\{\boldsymbol{w}_{b}^{\mathrm{BS}} \mid r_{b, u} \leq r_{\max }, r_{\max }=r_{c}\left(z_{b}, \Psi\right), \boldsymbol{w}_{b}^{\mathrm{BS}} \in \Phi_{\mathcal{B}}\right\} .
$$

In the latter, i.e., user with omni-directional antenna, the beamwidth is denoted by $\Psi_{0}$. The receiving coverage is the entire 3D space, and therefore $r_{\max }=\infty, \Phi_{\mathcal{C}}^{u}=\Phi_{\mathcal{B}}$, and the receive antenna gain is $G_{u}^{\mathrm{rx}}\left(\Psi_{0}\right)=1$. Throughout this paper, we assume TU is equipped with an omni-directional antenna. Hence, the set of BSs within the receiving coverage of the TU is $\Phi_{\mathcal{C}}^{t}=\Phi_{\mathcal{B}}$ and its receive antenna gain is $G_{t}^{\mathrm{rx}}\left(\Psi_{0}\right)=1$.

In addition, the antenna radiation pattern of the $B S$ is assumed to be horizontally omni-directional and vertically directional. As the antennas of the BSs are typically downtilted, $\mathrm{AU}$ and TU are assumed to receive signals from the BSs' sidelobe and mainlobe, respectively, as in [13]. For notation simplicity, the mainlobe and sidelobe gains of the BSs are considered as part of the channel gain as discussed in the sequel.

\section{Channel Model}

According to [8], [9], [13], [37], the aerial communication link between $\mathrm{AU}$ and the $\mathrm{BS} b$ follows a probabilistic LOS/non-LOS (NLOS) model. We denote $v \in\{\mathrm{L}, \mathrm{N}\}$ as the types of links, where, L, and, N, represent the LOS and NLOS links, respectively. The probability of LOS between the AU and $\mathrm{BS} b$ is expressed as:

$$
\mathcal{P}_{b, u}^{\mathrm{L}}\left(r_{b, u}, \triangle z_{u}\right)=-\varphi \cdot \exp \left(-\xi \cdot \phi_{b, u}^{\mathrm{ele}}\right)+\zeta,
$$

where $\phi_{b, u}^{\text {ele }}=\tan ^{-1}\left(\frac{\triangle z_{u}}{r_{b, u}}\right)$ is the elevation angle. In (3), $\varphi$, $\xi$ and $\zeta$ are constant coefficients related to the communication environment [37]. The probability of NLOS between AU and $\mathrm{BS} b$ is $\mathcal{P}_{b, u}^{\mathrm{N}}\left(r_{b, u}, \triangle z_{u}\right)=1-\mathcal{P}_{b, u}^{\mathrm{L}}\left(r_{b, u}, \triangle z_{u}\right)$.

Given link type, the channel gain between AU and BS $b$ is:

$$
\left|h_{b, u}^{v}\right|^{2}=\Xi_{b, u}^{v}\left|\Omega_{b, u}^{v}\right|^{2}, v \in\{\mathrm{L}, \mathrm{N}\},
$$

where $\Xi_{b, u}^{v} \triangleq \frac{A_{u}^{v} G_{u}^{\mathrm{rx}}(\Psi)}{d_{b, u}{ }^{\alpha v}}$ and $\left|\Omega_{b, u}^{v}\right|^{2}$ are the large and small-scale fading effects, respectively. Specifically, $A_{u}^{v}$ is the constant channel coefficient for $v$ link, $\alpha_{v}$ is the aerial path loss exponents, and $\left|\Omega_{b, u}^{v}\right|^{2} \sim \operatorname{Gamma}\left(m_{u}^{v}, \theta_{u}^{v}\right)$. Note that $A_{u}^{v}$ accounts for the sidelobe gain, fixed transmission power, excessive loss due to $v$ link, and propagation loss at a carrier 
frequency $f_{c}$. For tractability, $m_{u}^{v}$ is also assumed to be an integer as in [8], [9], [13], [37].

The channel gain between the TU and $\mathrm{BS} b$ is:

$$
\left|h_{b, t}\right|^{2}=\Xi_{b, t}\left|\Omega_{b, t}\right|^{2} .
$$

In (5), $\Xi_{b, t} \triangleq \frac{A_{t} G_{t}^{\mathrm{rx}}\left(\Psi_{0}\right)}{d_{b} t^{\alpha}}$ and $\left|\Omega_{b, t}\right|^{2}$ are the large and smallscale fading effects, respectively. In particular, $A_{t}$ is the attenuation for terrestrial link, $\alpha$ is the terrestrial path loss exponent, and $\left|\Omega_{b, t}\right|^{2}$ is exponentially distributed with unit mean. Here, $A_{t}$ accounts for the mainlobe gain, fixed transmission power, and propagation loss at a carrier frequency $f_{c}$.

\section{User Association}

Referring to [8], [9], we consider two types of user association policies, namely, minimum-distance and maximumSINR. In minimum-distance based user association, the AU is connected to the closest BS [8]. In particular, the associated BS is selected as follows:

$$
b_{0}=\left\{b \mid \min r_{b, u}, \forall b \in \Phi_{\mathcal{C}}^{u}\right\} .
$$

In maximum-SINR based user association, the AU is connected to the BS that provides the strongest (average) received signal [9]. Specifically, the associated BS is chosen as follows:

$$
b_{0}=\left\{\left.b|\max | \Xi_{b, u}^{v}\right|^{2}, \forall b \in \Phi_{\mathcal{C}}^{u}, v \in\{\mathrm{L}, \mathrm{N}\}\right\} .
$$

It is worth highlighting that, due to LOS/NLOS links, the associated BS using the maximum-SINR based user association may not be the nearest BS.

\section{E. Inter-Cell Interference Coordination}

To further mitigate the effect of strong ICI, we consider the application of ICIC. We suppose that an orthogonal RB, which is scheduled for the $\mathrm{AU}$, is being muted by $K-1$ neighboring BSs. In particular, we denote $\chi_{b}$ as the coordinated scheduling policy. We assume that BS $b$ is transmitting at that orthogonal $\mathrm{RB}$ if $\chi_{b}=1$, and BS $b$ is not transmitting if $\chi_{b}=0$. The exact value of $\chi_{b}$ is difficult to model in a PPP as the locations of the AU, interfering BSs and $\chi_{b}$ are correlated. A typical method to maintain tractability is to approximate the set of interfering BS by a PPP with an intensity of $\frac{\lambda}{K}$ (see, [38], [39]). Referring to [40], [41], we therefore model $\chi_{b}$ as an independent and identically distributed (i.i.d.) Bernoulli random variable of a mean of $\frac{1}{K}$. If $K>1$, then $K-1$ BSs are coordinated and for $K=1$, no coordination is needed.

\section{F. Non-Orthogonal Multiple Access}

The superimposed signal of AU and TU transmitted by their associated $\mathrm{BS} b_{0}$ is:

$$
S_{b_{0}}=\sqrt{\rho_{u}} S_{u}+\sqrt{\rho_{t}} S_{t},
$$

where $S_{u}$ and $S_{t}$ are the information bearing for $\mathrm{AU}$ and TU, respectively, with $\mathbb{E}\left[\left|S_{u}\right|^{2}\right]=\mathbb{E}\left[\left|S_{t}\right|^{2}\right]=1$. Furthermore, $\rho_{u}$ and $\rho_{t}$ are the power coefficients for AU and TU, respectively, where $\rho_{u}+\rho_{t}=1$. The received signal at $\mathrm{AU}$ is:

$$
y_{u}^{\mathrm{rx}}=h_{b_{0}, u} S_{b_{0}}+\sum_{b \in \Phi_{\mathcal{I}}^{u}} \chi_{b} h_{b, u} S_{b}+\sigma_{u},
$$

where $h_{b, u}$ is the complex channel gain from BS $b$ to the AU. In (9), the first term is the superimposed signal from the associated base station $b_{0}$, the second term is the ICI from $\Phi_{\mathcal{I}}^{u}=\Phi_{\mathcal{C}}^{u} /\left\{b_{0}\right\}$, and $\sigma_{u} \sim \mathrm{CN}\left(0, N_{0}\right)$ is the additive white Gaussian noise (AWGN) observed at the AU, where $N_{0}$ is the noise power level. The received signal at $\mathrm{TU}$ is:

$$
y_{t}^{\mathrm{rx}}=h_{b_{0}, t} S_{b_{0}}+\sum_{b \in \Phi_{\mathcal{I}}^{t}} \chi_{b} h_{b, t} S_{b}+\sigma_{t},
$$

where $\Phi_{\mathcal{I}}^{t}=\Phi_{B} /\left\{b_{0}\right\}$ and $\sigma_{t} \sim \mathrm{CN}\left(0, N_{0}\right)$ is AWGN observed at the TU.

To ensure a reliable operation, we assume AU decodes its message directly and its SINR is:

$$
\operatorname{SIN} R_{u}^{v}=\frac{\rho_{u}\left|h_{b_{0}, u}^{v}\right|^{2}}{\rho_{t}\left|h_{b_{0}, u}^{v}\right|^{2}+\sum_{b \in \Phi_{\mathcal{I}}^{u}} \chi_{b}\left|h_{b, u}^{v}\right|^{2}+N_{0}},
$$

where $\left|h_{b_{0}, u}^{v}\right|^{2}$ is the channel gain between AU and BS $b_{0}$. Consequently, TU has to perform the SIC by first removing the message of the AU with the following SINR:

$$
S I N R_{t}^{(u)}=\frac{\rho_{u}\left|h_{b_{0}, t}\right|^{2}}{\rho_{t}\left|h_{b_{0}, t}\right|^{2}+\sum_{b \in \Phi_{\mathcal{工}}^{t}} \chi_{b}\left|h_{b, t}\right|^{2}+N_{0}},
$$

where $\left|h_{b_{0}, t}\right|^{2}$ is the channel gain between TU and BS $b_{0}$. Here, we assume perfect SIC, i.e., the message of the AU can be perfectly removed from the superimposed signal if $S I N R_{t}^{(u)} \geq T_{\min }$, where $T_{\min }$ is the targeted SINR required to decode the AU's message. TU then decodes its own message with the following SINR:

$$
S I N R_{t}=\frac{\rho_{t}\left|h_{b_{0}, t}\right|^{2}}{\sum_{b \in \Phi_{\mathcal{I}}^{t}} \chi_{b}\left|h_{b, t}\right|^{2}+N_{0}} .
$$

Note that we have considered the reverse SIC order, i.e., TU decodes the signal directly and AU performs SIC, in prior. Our sequel analysis can also be easily extended to those cases. Nevertheless, due to ICI, it does not provide an efficient performance to the applications focused in this paper. Therefore, for brevity, we omit its discussions.

\section{G. Coverage probability}

The coverage probability of the AU is defined as:

$$
\mathcal{P}_{\text {cov }, u}(T)=\mathcal{P}\left(S I N R_{u}>T\right),
$$

where $S I N R_{u}$ is the SINR of the AU regardless of the link type, $v$, and $T$ is a SINR threshold. Note that the TU needs to remove the AU's message to decode its own message. Therefore, the coverage probability of the TU is:

$$
\mathcal{P}_{\text {cov }, t}(T)=\mathcal{P}\left(S I N R_{t}^{(u)}>T_{\min }, S I N R_{t}>T\right),
$$

where $T_{\min }=2^{\frac{R_{\min }}{B_{\mathrm{w}}}}-1, R_{\min }$ and $B_{\mathrm{w}}$ are the targeted data rates required to successfully decode the AU's message, and the system bandwidth, respectively. The coverage probability 
can be equivalently thought as the probability of a randomly chosen user that can achieve SINR $T$, or the average fraction of users that at any time achieve SINR $T$ [38].

\section{PERFORMANCE ANALYSIS}

In this section, we analyze the performance of AU and TU. We first present the distribution of $r_{u}$ and $r_{t}$. Then, we derive the coverage probability and average rate of AU and TU.

\section{A. Distribution of $r_{u}$ and $r_{t}$}

To derive the coverage probability of AU, we first obtain the distribution of $r_{u}$. The following lemma provides the general distribution of $r_{u}$ in the proposed framework.

Lemma 1. If a minimum-distance or maximum-SINR based user association is employed, the distribution of $r_{u}$ is:

$$
f_{r_{u}}\left(r_{0}\right)=\sum_{\forall v} P_{u}^{v} F_{p, r_{u}}^{v}\left(r_{\max }\right) \hat{f}_{p, r_{u}}^{v}\left(r_{0}\right)
$$

and the conditional distribution of $r_{u}$ is:

$$
\hat{f}_{p, r_{u}}^{v}\left(r_{0}\right)=\frac{\mathbf{1}_{r_{0} \leq r_{\max }}\left\{f_{r_{u}}^{v}\left(r_{0}\right)\right\}}{F_{p, r_{u}}^{v}\left(r_{\max }\right)},
$$

where $f_{r_{u}}^{v}\left(r_{0}\right)$ for minimum-distance and maximum-SINR based user association are respectively given in (18) and (22), $P_{u}^{v}$ for minimum-distance and maximum-SINR based user association are respectively given in (20) and (28), and $F_{p, r_{u}}^{v}\left(r_{\max }\right)=\int_{0}^{r_{\max }} f_{p, r_{u}}^{v}\left(r_{0}\right) d r_{0}$.

Proof: According to [25], if a minimum-distance based user association is employed, the unconditional probability density function (pdf) of $r_{u}$ is $f_{r_{u}}\left(r_{0}\right)=$ $2 \pi \lambda r_{0} \exp \left(-\lambda \pi r_{0}^{2}\right)$. Conditioning on $v$, the conditional pdf of $r_{u}$ is derived as:

$$
f_{r_{u}}^{v}\left(r_{0}\right)=\frac{B^{v} f_{p, r_{u}}^{v}\left(r_{0}\right)}{P_{u}^{v}}
$$

where

$$
\begin{aligned}
f_{p, r_{u}}^{v}\left(r_{0}\right) & =\frac{2 \pi \lambda \mathcal{P}_{b, u}^{v}\left(r_{0}, \triangle z_{u}\right) r_{0} \exp \left(-\lambda \pi r_{0}^{2}\right)}{B^{v}} \\
P_{u}^{v} & =\int_{0}^{r_{\max }} B^{v} f_{p, r_{u}}^{v}\left(r_{0}\right) d r_{0} \\
B^{v} & =1-\exp \left(-2 \lambda \pi \int_{0}^{r_{\max }} \mathcal{P}_{b, u}^{v}\left(r_{0}, \triangle z_{u}\right) r_{0} d r_{0}\right) .
\end{aligned}
$$

Specifically, (19) is a pdf for $v$ link, (20) is the probability that $\mathrm{AU}$ is connected to $\mathrm{BS} b_{0}$ with a link type $v,(21)$ is the probability that having at least one BS with $v$ link in the PPP. Furthermore, $R^{v}\left(r_{0}\right)=r_{0}, \forall v$.

For maximum-SINR based user association, the conditional distribution of $r_{u}$ is obtained using a similar argument as in [42], Lemma 1-3. The conditional pdf of $r_{u}$ being served by link type $v$ is:

$$
f_{r_{u}}^{v}\left(r_{0}\right)=\frac{B^{v} f_{p, r_{u}}^{v}\left(r_{0}\right) \exp \left(\mathcal{I}_{v^{\prime}}^{v}\left(r_{0}\right)\right)}{P_{u}^{v}}
$$

where

$$
\begin{aligned}
f_{p, r_{u}}^{v}\left(r_{0}\right) & =\frac{2 \pi \lambda \mathcal{P}_{b, u}^{v}\left(r_{0}, \Delta z_{u}\right) r_{0} \exp \left(\mathcal{I}_{v}^{v}\left(r_{0}\right)\right)}{B^{v}}, \\
\mathcal{I}_{v}^{v}\left(r_{0}\right) & =-2 \lambda \pi \int_{0}^{r_{0}} \mathcal{P}_{b, u}^{v}\left(r_{i}, \Delta z_{u}\right) r_{i} d r_{i}, \\
\mathcal{I}_{v^{\prime}}^{v}\left(r_{0}\right) & =-2 \lambda \pi \int_{0}^{R^{v}\left(r_{0}\right)} \mathcal{P}_{b, u}^{v^{\prime}}\left(r_{i}, \Delta z_{u}\right) r_{i} d r_{i}, \\
R^{\mathrm{L}}\left(r_{0}\right) & =\sqrt{\left[\left(\frac{A_{u}^{\mathrm{N}}}{A_{u}^{\mathrm{L}}}\right)^{\frac{2}{\alpha_{\mathrm{N}}}}\left(r_{0}^{2}+\Delta z_{u}^{2}\right)^{\frac{\alpha_{\mathrm{L}}}{\alpha_{\mathrm{N}}}}-\Delta z_{u}^{2}\right]_{0}^{+}}, \\
R^{\mathrm{N}}\left(r_{0}\right) & =\sqrt{\left[\left(\frac{A_{u}^{\mathrm{L}}}{A_{u}^{\mathrm{N}}}\right)^{\frac{2}{\alpha_{\mathrm{L}}}}\left(r_{0}^{2}+\Delta z_{u}^{2}\right)^{\frac{\alpha_{\mathrm{N}}}{\alpha_{\mathrm{L}}}}-\Delta z_{u}^{2}\right]_{r_{\max }^{2}}^{-}}, \\
P_{u}^{v} & =\int_{0}^{r_{\max }} B^{v} \exp \left(\mathcal{I}_{v^{\prime}}^{v}\left(r_{0}\right)\right) f_{p, r_{u}}^{v}\left(r_{0}\right) d r_{0},
\end{aligned}
$$

and $v^{\prime}=\{\mathrm{L}, \mathrm{N}\} \backslash v$. In particular, (23) is the pdf for $v$ link, (24) is the probability of having no BS with $v$ link stronger than that of $\mathrm{BS} b_{0},(25)$ is the probability of having no BS with $v^{\prime}$ link stronger than that of BS $b_{0}$. Furthermore, (26) and (27) are the maximum and minimum distances for (25) to hold true, and (28) is the probability that the AU is associated to BS $b_{0}$ with $v$ link. By truncating the domain of (18)-(21) and (22)-(28), we obtain (16-17).

To obtain the coverage probability of TU, the distribution of $r_{t}$ is given in Lemma 2.

Lemma 2. The conditional pdf of $r_{t}$ given $r_{u}$ is:

$$
f_{r_{t} \mid r_{u}}\left(r_{2}\right)=\frac{2}{\gamma} f_{r \mid r_{u}}\left(\frac{2 r_{2}}{\gamma}\right)
$$

where

$$
\begin{array}{r}
f_{r \mid r_{u}}\left(r_{1}\right)= \begin{cases}\lambda k_{1} \exp \{-\lambda k\}, & r_{1} \leq 2 r_{0}, \\
2 \lambda \pi r_{1} \exp \left\{-\lambda \pi\left(r_{1}^{2}-r_{0}^{2}\right)\right\}, & r_{1}>2 r_{0},\end{cases} \\
k=\pi r_{1}^{2}-\sum_{i=0}^{1}\left(r_{i}^{2} \cos ^{-1}\left(\frac{c_{i}}{r_{i}}\right)-c_{i} \sqrt{r_{i}^{2}-c_{i}^{2}}\right), \\
c_{0}=\frac{2 r_{0}^{2}-r_{1}^{2}}{2 r_{0}}, c_{1}=\frac{r_{1}^{2}}{2 r_{0}}, \text { and } k_{1}=2 \pi r_{1}-2 r_{1} \cos ^{-1}\left(\frac{r_{1}}{2 r_{0}}\right) .
\end{array}
$$

Proof: See Appendix A.

Depending on the user association policy, the coverage probability is obtained using the corresponding unconditional/conditional pdf. In the sequel, we focus on the conditional coverage probability of the users without repeatedly discussing the distribution of $r_{u}$ and $r_{t}$.

\section{B. AU's Coverage Probability}

Theorem 1. Conditioning on $r_{u}$ and $v$, the downlink coverage probability of a typical AU using NOMA is:

$$
\begin{aligned}
\mathcal{P}_{\text {cov }, u}(T)= & \int_{0}^{r_{\max }} \sum_{\forall v} \mathcal{P}\left(S I N R_{u}^{v}>T \mid r_{u}\right) \times \\
& P_{u}^{v} F_{p, r_{u}}^{v}\left(r_{\max }\right) \hat{f}_{r_{u}}^{v}\left(r_{0}\right) d r_{0},
\end{aligned}
$$


where

$$
\begin{aligned}
\mathcal{P}\left(S I N R_{u}^{v}>T \mid r_{u}\right) & =\sum_{i=0}^{m_{u}^{v}-1}(-1)^{i} s_{i}^{v} \frac{\partial^{i}}{\partial q_{v}^{i}} \mathcal{L}^{v} I_{u}\left(q_{v}\right), \\
s_{i}^{v} & =\frac{\exp \left(-q_{v} N_{0}\right)}{i !} \sum_{j=i}^{m_{u}^{v}-1} \frac{N_{0}^{j-i} q_{v}^{j}}{(j-i) !}, \\
q_{v} & =\frac{T}{\left(\rho_{u}-\rho_{t} T\right) A_{u}^{v} G_{u}^{r x}(\Psi)\left(r_{u}^{2}+\Delta z_{u}^{2}\right)^{-\frac{\alpha_{u}^{v}}{2}} \theta_{u}^{v}}, \\
\mathcal{L}_{I_{u}}^{v}\left(q_{v}\right)= & \exp \left(\sum_{\forall \tilde{v}} \int_{R^{v}, \tilde{v}\left(r_{u}\right)}^{r_{\max }}-2 \pi \frac{\lambda}{K} \times\right. \\
g^{\tilde{v}}\left(q_{v}\right)= & \left(\begin{array}{ll}
1+q_{v} A_{u}^{\tilde{v}} G_{u}^{r x}(\Psi)\left(r_{u}^{2}+\Delta z_{u}^{2}\right)^{-\frac{\alpha_{u}^{\tilde{u}}}{2}} \theta_{u}^{\tilde{v}}
\end{array}\right), \\
R^{v, \tilde{v}}\left(r_{u}\right)= & \begin{cases}r_{u}, & v=\tilde{v}, \\
R^{v}\left(r_{0}\right), & v \neq \tilde{v} .\end{cases}
\end{aligned}
$$

Proof: See Appendix B.

It is rather difficult to directly compute the derivative of the Laplace transform of interference at AU in (30). To address this issue, we further provide the following lemma to further simplify its computation.

Lemma 3. The $i^{\text {th }}$ derivative of the $\mathcal{L}^{v}{ }_{I_{u}}\left(q_{v}\right)$ is:

$$
\frac{\partial^{i}}{\partial q_{v}^{i}} \mathcal{L}^{v}{ }_{I_{u}}\left(q_{v}\right)=\mathcal{L}^{v}{ }_{I_{u}}\left(q_{v}\right) B_{i}\left(\mathcal{J}^{(1)}\left(q_{v}\right), \mathcal{J}^{(2)}\left(q_{v}\right), \ldots, \mathcal{J}^{(i)}\left(q_{v}\right)\right),
$$

where $B_{i}(\cdot)$ is the complete Bell polynomial [43] and

$$
\begin{aligned}
\mathcal{J}^{(j)}\left(q_{v}\right)= & \sum_{\forall \tilde{v}}\left[\int_{R^{v, \tilde{v}}\left(r_{u}\right)}^{r_{\max }} \frac{\partial^{j}}{\partial q_{v}^{j}}-2 \pi \frac{\lambda}{K} \times\right. \\
& \left.\mathcal{P}_{b, u}^{\tilde{v}}\left(r_{b, u}, \Delta z_{u}\right)\left(1-g^{\tilde{v}}\left(q_{v}\right)\right) r_{b, u} d r_{b, u}\right] .
\end{aligned}
$$

Proof: See Appendix C.

\section{TU's Coverage Probability}

Next, we analyze the performance of $\mathrm{TU}^{1}$. Note that the performance of TU is generally more complicated than that of $\mathrm{AU}$ as the location of TU depends on $b_{0}$ and $b_{1}$. Furthermore, the location of TU introduces non-symmetric property. These make the analysis very challenging and therefore, in this paper, a significant effort has been made to simplifying many complicated expressions to make it computation feasible.

To obtain the exact coverage probability of TU, we first derive the Laplace transform of interference at TU, $\mathcal{L}_{I_{t}}\left(q_{t}\right)$. For brevity of expositions, we write the aggregated interference of TU as:

$$
I_{t}=\chi_{b_{1}}\left|h_{b_{1}, t}\right|^{2}+\sum_{b \in \Phi_{I}^{t} /\left\{b_{1}\right\}} \chi_{b}\left|h_{b, t}\right|^{2}
$$

\footnotetext{
${ }^{1}$ Note that, here, angle is always referred to the azimuth angle of $b_{0}$ from the origin. E.g., $\tan _{2}^{-1}\left(y_{b_{0}}, x_{b_{0}}\right)=0$, where $\tan _{2}^{-1}$ denotes fourth quadrant inverse tangent.
}

In (32), the first term accounts for the dominant interference from $b_{1}$, and the second term accounts for the interference from other base stations, which forms a PPP outside $D_{o}$ and $D_{1}$ (see, Fig. 10 in Appendix A). Therefore, the Laplace transform of interference at $\mathrm{TU}$ is written as:

$$
\mathcal{L}_{I_{t}}\left(q_{t}\right)=\mathcal{L}_{b_{1}}\left(q_{t}\right) \mathcal{L}_{\Phi}\left(q_{t}\right),
$$

Lemma 4. The Laplace transform of dominant interference at $T U$, i.e. interference from $B S b_{1}$, is:

$$
\begin{aligned}
\mathcal{L}_{b_{1}}\left(q_{t}\right)= & \left(1-\frac{1}{K}\right)+\frac{1}{K\left(\Theta^{+}-\Theta^{-}\right)} \times \\
& \int_{\Theta^{-}}^{\Theta^{+}} \frac{1}{1+q_{t} A_{t}\left(l_{b_{1}}\left(\phi_{i, t}\right)^{2}+\triangle z_{t}^{2}\right)^{-\frac{\alpha}{2}}} d \phi_{i, t},
\end{aligned}
$$

where $l_{b_{1}}\left(\phi_{i, t}\right)=\sqrt{r_{1}^{2}+r_{t}^{2}-2 r_{1} r_{t} \cos \left(\theta_{t}-\phi_{i, t}\right)}$, and

$$
\begin{gathered}
\Theta^{+}=\left\{\begin{array}{ll}
\Theta, & r_{1} \leq 2 r_{0}, \\
2 \pi, & r_{1}>2 r_{0},
\end{array} \Theta^{-}= \begin{cases}-\Theta & r_{1} \leq 2 r_{0}, \\
0 & r_{1}>2 r_{0},\end{cases} \right. \\
\Theta=\pi-\cos ^{-1}\left(\frac{r_{1}}{2 r_{0}}\right) .
\end{gathered}
$$

Proof: (34) is obtained by using the complementary cumulative distribution function (ccdf) and moment generating function (MGF) of $\left|\Omega_{b_{1}, t}\right|^{2}$ and $\chi_{b_{1}}$. Meanwhile, $l_{b_{1}}\left(\phi_{i, t}\right)$ is derived using the law of cosines and the fact that $b_{1}$ is uniformly distributed between the angle $\Theta^{-}$and $\Theta^{+}$.

Lemma 5. The Laplace transform of PPP interference at $T U$, i.e., interference from $B S s$ outside $D_{o}$ and $D_{1}$, is:

$$
\begin{aligned}
\mathcal{L}_{\Phi}\left(q_{t}\right)= & \exp \left(-\frac{\lambda}{K} \frac{\left(q_{t} A_{t}\right)^{\frac{2}{\alpha}}}{\alpha} \times\right. \\
& \left.\int_{0}^{2 \pi} \bar{B}\left(\left[1+q_{t} A_{t}\left(l\left(\phi_{i, t}\right)^{2}+\triangle z_{t}^{2}\right)^{-\frac{\alpha}{2}}\right]^{-1}, \frac{2}{\alpha}, 1-\frac{2}{\alpha}\right) d \phi_{i, t}\right),
\end{aligned}
$$

where $\bar{B}(z, x, y)$ is the Beta upper incomplete function, and

$$
\begin{aligned}
l\left(\phi_{i, t}\right) & = \begin{cases}l_{D}\left(\phi_{i, t}\right), & r_{1} \leq 2 r_{0}, \\
r_{t} \cos \left(\tilde{\theta}_{i, 1}\right)+\sqrt{r_{1}^{2}-r_{t}^{2} \sin ^{2}\left(\tilde{\theta}_{i, 1}\right)}, & r_{1}>2 r_{0},\end{cases} \\
l_{D}\left(\phi_{i, t}\right) & = \begin{cases}r_{t} \cos \left(\tilde{\theta}_{i, 1}\right)+\sqrt{r_{1}^{2}-r_{t}^{2} \sin ^{2}\left(\tilde{\theta}_{i, 1}\right)}, & \Upsilon^{-} \leq \phi_{i, t} \leq \Upsilon^{+}, \\
\sqrt{l_{i, 1}^{2}+r_{t}^{2}-\left(2 l_{i, 1} r_{t} \cos \left(\theta_{t}-\theta_{i, 1}\right)\right)}, & \text { otherwise, }\end{cases} \\
\theta_{i, 1} & = \begin{cases}\pi-\arccos \left(\frac{l_{i, 1}}{2 r_{0}}\right), & \phi_{i, t} \leq \Upsilon, \\
\pi+\arccos \left(\frac{l_{i, 1}}{2 r_{0}}\right), & \text { otherwise, }\end{cases} \\
\tilde{\theta}_{i, 1} & =\pi-\theta_{t}+\phi_{i, t}, \\
l_{i, 1} & =\sqrt{2 r_{0}^{2}\left(1-\cos \left(\theta_{i, 0}\right)\right),} \\
\Upsilon & =\tan _{2}^{-1}\left(-r_{t} \sin \left(\theta_{t}\right),-2 r_{0}-r_{t} \cos \left(\theta_{t}\right)\right), \\
\Upsilon^{ \pm} & =\tan _{2}^{-1}\left(r_{1} \sin ( \pm \Theta)-r_{t} \sin \left(\theta_{t}\right), r_{1} \cos ( \pm \Theta)-r_{t} \cos \left(\theta_{t}\right)\right), \\
\theta_{i, 0} & = \begin{cases}\tan _{2}^{-1}\left(s_{3}, s_{2}+s_{1} s_{3}\right), & \phi_{i, t} \leq \pi, \\
\tan _{2}^{-1}\left(s_{4}, s_{2}+s_{1} s_{4}\right), & \text { otherwise, },\end{cases}
\end{aligned}
$$




$$
\begin{array}{ll}
s_{1}=\frac{1}{\tan \left(\phi_{i, t}\right)}, & s_{2}=\frac{r_{0}+r_{t} \cos \left(\theta_{t}\right)}{r_{0}}-\frac{r_{t} \sin \left(\theta_{t}\right)}{r_{0} \tan \left(\phi_{i, t}\right)}, \\
s_{3}=\frac{-s_{1} s_{2}+\sqrt{1+s_{1}^{2}-s_{2}^{2}}}{1+s_{1}^{2}}, s_{4}=\frac{-s_{1} s_{2}-\sqrt{1+s_{1}^{2}-s_{2}^{2}}}{1+s_{1}^{2}} .
\end{array}
$$

Proof: (35) is obtained by using the ccdf, MGF of $\left|\Omega_{b, t}\right|^{2}$, and the probability generating functional (PGFL). The key challenge is to derive $l\left(\phi_{i, t}\right)$. As seen in Fig. 10, Appendix $\mathrm{A}$, there are two cases to be considered: (i) $r_{1} \leq 2 r_{0}$, and (ii) $r_{1}>2 r_{0}$. For case (ii), we can obtain $l\left(\phi_{i, t}\right)$ using law of cosines. Similarly, for case (i), we can derive $l_{D}\left(\phi_{i, t}\right)$ using law of cosines if $\Upsilon^{-} \leq \phi_{i, t} \leq \Upsilon^{+}$. Nevertheless, given $\phi_{i, t}$, where $\Upsilon^{+} \leq \phi_{i, t} \leq \Upsilon^{-}$, we have to solve for $\theta_{i, 0}$, where

$$
\cos \left(\theta_{i, 0}\right)-\frac{\sin \left(\theta_{i, 0}\right)}{\tan \left(\phi_{i, t}\right)}=\frac{r_{0}+r_{t} \cos \left(\theta_{t}\right)}{r_{0}}-\frac{r_{t} \sin \left(\theta_{t}\right)}{r_{0} \tan \left(\phi_{i, t}\right)} \text {. }
$$

Formulating it as a quadratic expression and noting $\Upsilon^{+} \leq$ $\phi_{i, t} \leq \Upsilon^{-}$, we then obtain $\theta_{i, 0}$ in a closed-form expression as expressed above.

Given the distribution of $r_{u}, r_{t}$ and Laplace transform of interference, $\mathcal{L}_{I}\left(q_{t}\right)$, now we can obtain the coverage probability of TU.

Theorem 2. The downlink coverage probability of TU being at a distance of $r_{t}$ away from BS $b_{0}$ using NOMA is:

$\mathcal{P}_{\text {cov }, t}(T)=$

$\int_{0}^{2 \pi} \int_{0}^{\infty} \int_{0}^{\infty} \mathcal{P}\left(S I N R_{t}^{(u)}>T_{\min }, S I N R_{t}>T \mid \theta_{t}, r_{t}, r_{u}\right)$

$\times \frac{1}{2 \pi} f_{r_{t} \mid r_{u}}\left(r_{2}\right) f_{r_{u}}\left(r_{0}\right) d r_{2} d r_{0} d \theta_{t}$,

where

$$
\begin{aligned}
& \mathcal{P}\left(\operatorname{SIN}_{t}^{(u)}>T_{\min }, \operatorname{SIN} R_{t}>T \mid \theta_{t}, r_{t}, r_{u}\right)=\exp \left(-q_{t} N_{0}\right) \mathcal{L}_{I_{t}}\left(q_{t}\right), \\
& q_{t}=\max \left(\frac{T}{\rho_{t} A_{t}\left(r_{t}^{2}+\Delta z_{t}^{2}\right)^{-\frac{\alpha}{2}}}, \frac{T_{\min }}{\left(\rho_{u}-\rho_{t} T_{\min }\right) A_{t}\left(r_{t}^{2}+\Delta z_{t}^{2}\right)^{-\frac{\alpha}{2}}}\right) .
\end{aligned}
$$

Proof: See Appendix D.

\section{Average Rate of $A U$ and $T U$}

Given the coverage probabilities of the users, the average rates of the users is:

$$
\begin{aligned}
\mathcal{R} & =\mathbb{E}\left[B_{\mathrm{w}} \log _{2}(1+\operatorname{SINR})\right] \\
& =\frac{B_{\mathrm{w}}}{\ln 2} \int_{0}^{\infty} \frac{\mathcal{P}_{\text {cov }}(T)}{1+T} d T,
\end{aligned}
$$

where $B_{\mathrm{w}}$ is the bandwidth of the system, SINR is the SINR of the users, and $\mathcal{P}_{\text {cov }}(T)$ is the coverage probabilities of the users. For example, $\mathcal{P}_{\text {cov }}(T)$ of AU is $\mathcal{P}_{\text {cov }, u}(T)$ and $\mathcal{P}_{\text {cov }}(T)$ of TU is $\mathcal{P}_{\text {cov }, t}(T)$. According to [13], [44], (37) can also be approximated using the Gauss-Chebyshev Quadrature as:

$$
\mathcal{R} \approx \frac{B_{\mathrm{w}}}{\ln 2} \sum_{n=1}^{N} \frac{\mathcal{P}_{\mathrm{cov}}\left(T_{n}\right)}{1+T_{n}} \cdot \frac{\pi^{2} \sin \left(\frac{2 n-1}{2 N} \pi\right)}{4 N \cos ^{2}\left[\frac{\pi}{4} \cos \left(\frac{2 n-1}{2 N} \pi\right)+\frac{\pi}{4}\right]},
$$

where $N$ is a parameter to be adjusted to ensure high accuracy and

$$
T_{n}=\tan \left[\frac{\pi}{4} \cos \left(\frac{2 n-1}{2 N} \pi\right)+\frac{\pi}{4}\right] .
$$

As it is seen, (37) is sufficient but the approximation in (38) helps to eliminate computing an additional integral.
Table I: Simulation Parameters

\begin{tabular}{|c|c|}
\hline Parameter & Value \\
\hline$\rho_{u}, \rho_{t}$ & $0.9,0.1$ \\
\hline$(\varphi, \xi, \zeta)$ & $(1,0.151,1)$ \\
\hline$\left(\alpha_{u}^{\mathrm{L}}, \alpha_{u}^{\mathrm{N}}, \alpha_{t}\right)$ & $(2.5,3.5,3.5)$ \\
\hline$\left(A_{u}^{\mathrm{L}}, A_{u}^{\mathrm{N}}, A_{t}\right)$ & $(-36.4,-45.4,-32.4) \mathrm{dB}$ \\
\hline$T_{\min } / R_{\min }$ & $-3.2815 \mathrm{~dB} / 100 \mathrm{Kbps}$ \\
\hline User Association & Minimum-distance \\
\hline$\lambda$ & $10 \mathrm{BSs} / \mathrm{Km}^{2}$ \\
\hline$\left(z_{u}, z_{b}, z_{t}\right)$ & $(120,19,1.5) \mathrm{m}$ \\
\hline$\left(m_{u}^{\mathrm{L}}, m_{u}^{\mathrm{N}}, m_{t}\right)$ & $(3,1,1)$ \\
\hline$\left(\theta_{u}^{\mathrm{L}}, \theta_{u}^{\mathrm{N}}, \theta_{t}\right)$ & $\left(\frac{1}{3}, 1,1\right)$ \\
\hline$(\gamma, K)$ & $(1,1)$ \\
\hline Antenna type & Omni-directional \\
\hline
\end{tabular}

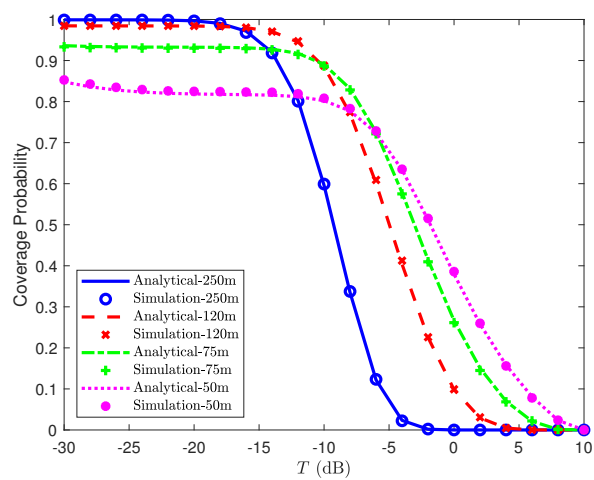

Figure 2: Coverage probabilities of the $\mathrm{AU}$ versus $T$ for different values of the AU's altitudes.

\section{Simulation and Analytical Results}

In this section, simulation and analytical results are presented to investigate the performance achieved by the proposed framework. We first examine the effect of different network parameters on the coverage probabilities and average rates achieved by $\mathrm{AU}$ and $\mathrm{TU}$. We then present an interference-aware downlink NOMA scheme for AU and TU. Unless stated otherwise, we use the simulation parameters as listed in Table I, which are based on [13], [37]. Note that $T_{\min }$ is obtained according the minimum rate required by $\mathrm{AU}$ for C\&C link as in [4].

Fig. 2 shows the coverage probabilities of AU for different values of AU's altitudes. As it is seen, our analytical results match the simulation results closely, which verifies the accuracy of (30)-(31). Using (30)-(31), we find that AU's altitude plays an important role in determining the coverage probability. In alignment with previous studies, a lower altitude leads to a higher coverage probability for a higher value of $T$. This is due to a weaker LOS ICI. Nevertheless, unlike the previous works, we would like to highlight that increasing AU's altitude is not always detrimental. This is because a higher altitude also improves the desired signal strength due to higher probability of LOS. Due to this factor, a higher altitude leads to a higher coverage probability for a lower $T$. Therefore, one may actually determine the AU's altitude based on their applications and ICI level.

Fig. 3 shows the impact of TU distance. As seen, our analytical results match the simulation results closely, which 


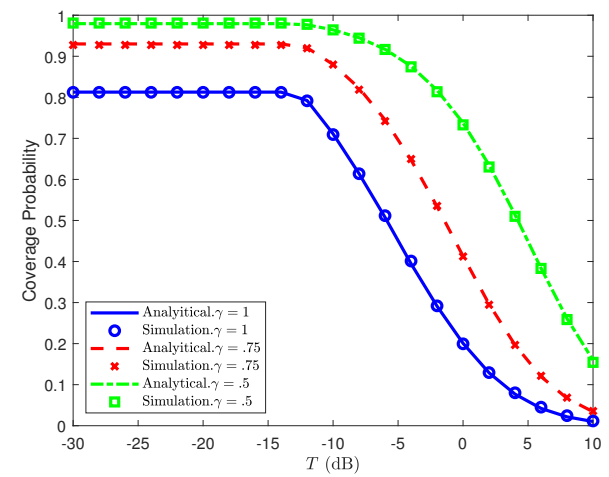

Figure 3: Coverage probabilities of the TU versus $T$ for different values of the TU's distances.

verifies the accuracy of (34)-(36). Using (36), we find that user association policy of the AU does not affect the performance of TUs. This is because the location of TU only depends on the location of $b_{0}$ and $b_{1}$. Besides, using (34) and (35), we also find that the ICI from BS $b_{1}$ alone is weaker than all other BSs in the networks. Furthermore, decreasing $\gamma$ generally improves the TU's SINR as shorter $r_{t}$ leads to a higher desired signal and a lower ICI level. Therefore, one may want to pair a near TU with a typical AU to obtain a higher rate.

Fig. 4 shows the impact of different power coefficients without SIC constraints (i.e., $T_{\min }=0$ ). Specifically, Fig. 4(a) presents the coverage probability of AU, Fig. 4(b) shows the coverage probability of TU, and Fig. 4(c) shows the (average) rate region between $\mathrm{AU}$ and TU. From these results, we can observe that, if $\rho_{u}$ increases, AU's coverage probability increases and TU's coverage probability decreases. In contrast, if $\rho_{t}$ increases, the opposite happens. This tradeoff is inevitable due to the standard rate region between $\mathrm{AU}$ and TU. Nevertheless, the coverage probability of the TU and rate region from these results are not sufficiently accurate as TU might have a weaker channel than that of $\mathrm{AU}$ due to deep fading or ICI. To ensure successful SIC at TU, SIC constraints must be imposed (i.e., $T_{\min }>0$ ).

In Fig. 5, we further consider the impact of SIC constraints. In Figs. 5(a) and 5(b), the impact of different targeted SINR, $T_{\min }$, and power coefficients, $\rho_{t}$, are investigated, respectively. Fig. 5(c) presents the new rate region between AU and TU with SIC constraints. Note that the coverage probability of AU remains unchanged and thus it is omitted. When SIC constraints are imposed, we find that some power coefficients (e.g., $\rho_{t} \geq 0.7$ ) lead to unsuccessful SIC as the co-channel interference is too strong. Furthermore, there exists an optimal power coefficient that maximizes the TU rate. Nonetheless, this optimal power coefficient does not maximize the AU's coverage probability as tradeoff still exists. This suggests that one might want to employ optimal power allocation scheme to obtain a higher TU rate without significantly degrading the AU's coverage probability.

Fig. 6 presents the impact of different user association policies. Our results show that maximum-SINR based user association is always more superior than minimum-distance

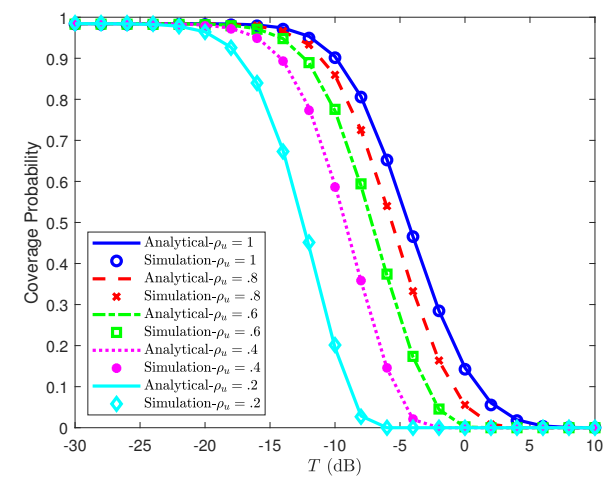

(a)

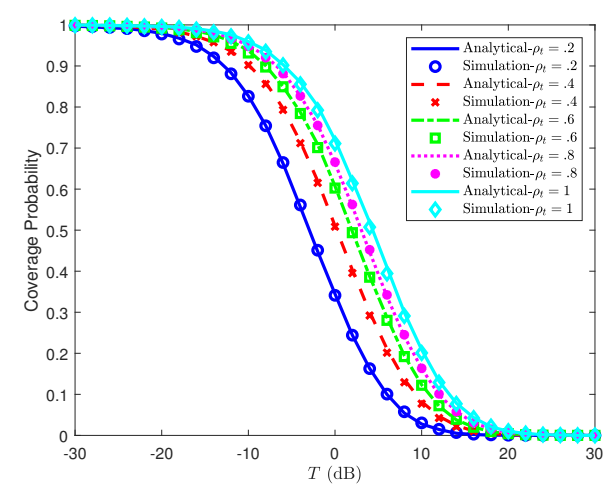

(b)

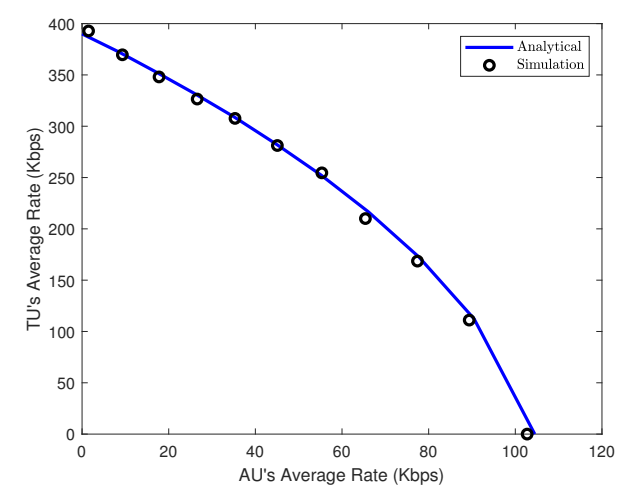

(c)

Figure 4: The impact of different power coefficients: a) coverage probabilities of the $\mathrm{AU} ; \mathrm{b}$ ) coverage probabilities of the TU; c) the rate region.

based user association. The gain is also more significant at a lower altitude. This is because, at a lower altitude, the probability of LOS is low. Therefore, ICI is weaker and connecting the AU to a BS with the strongest link significantly improves its desired signal. Nevertheless, at a higher altitude, AU suffers from many LOS ICI and therefore connecting AU to the strongest BS does not have a huge impact on improving its coverage probability.

Fig. 7 shows the impact of beamwidth, $\Psi$. As observed, there exists an optimal beamwidth that provides the highest coverage probability. This is because, if the beamwidth is too 


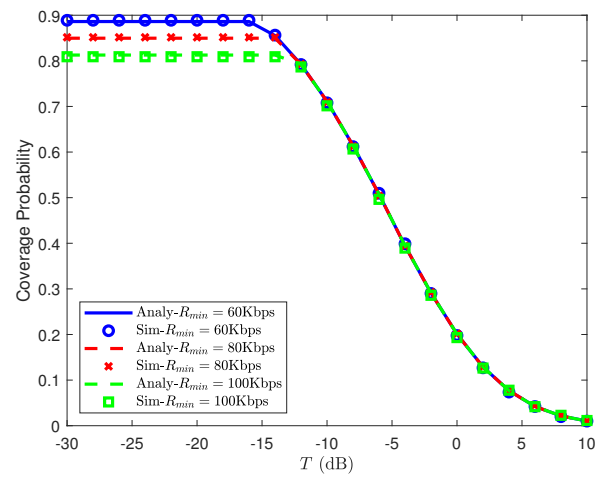

(a)

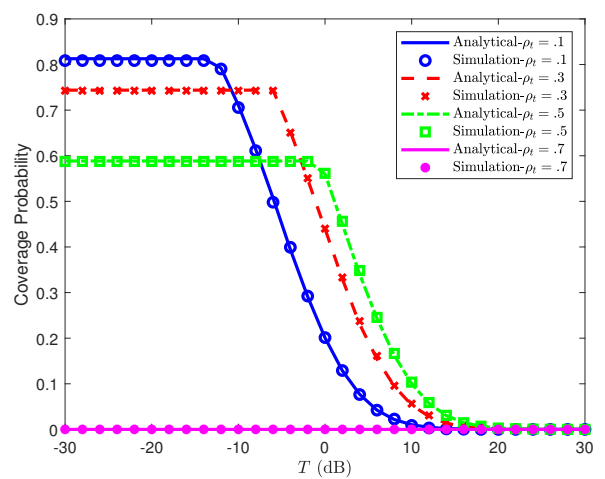

(b)

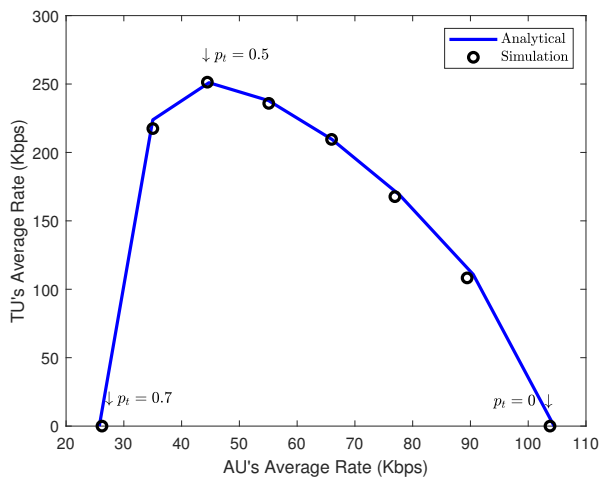

(c)

Figure 5: The impact of SIC constraints: a) coverage probabilities of the $\mathrm{TU}$ for different $\left.T_{\min } / R_{\min } ; \mathrm{b}\right)$ coverage probabilities of the $\mathrm{TU}$ for different $\rho_{t}$; c) the rate region.

small, there might not be a BS within the AU receiving coverage and, if the beamwidth is too large, the ICI becomes too strong. To avoid this fundamental tradeoff between coverage and ICI level, one may implement a re-configurable antenna at the AU. A re-configurable antenna allows AU to adaptively adjust its beamwidth. Therefore, it can significantly improve the AU's coverage probability but it comes with a higher implementation cost.

Fig. 8 depicts the impact of different number of coordinated BSs, $K$. In general, the coverage probability of AU increases as $K$ increases. However, its gain diminishes as $K$ becomes

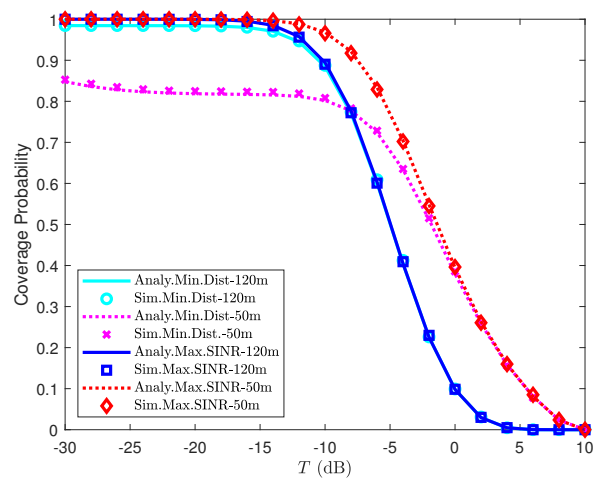

Figure 6: Coverage probabilities of the AU versus $T$ using different user association policies and values of the AU's altitudes.

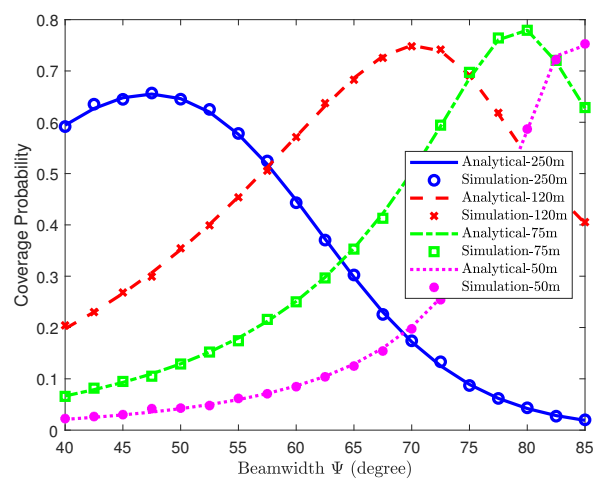

Figure 7: Coverage probabilities of the $\mathrm{AU}$ versus antenna beamwidth for different values of the AU's altitudes.

large. As compared to other strategies, such as maximumSINR based user association or directional antenna with fixed beamwidth, ICIC is generally a more effective strategy in NOMA. For example, using ICIC with $K=4$, the AU's coverage probability $(120 \mathrm{~m})$ improves up to 0.875 at $-3.2815 \mathrm{~dB}$. Meanwhile, maximum-SINR based user association and directional antenna only achieves 0.34 and 0.75 , respectively. Importantly, only ICIC improves the average rate of TU to 497Kbps. However, the key concerns of ICIC are its backhaul overhead, and system complexity.

These results suggest that an efficient usage of NOMA for cellular-connected UAVs requires a well-designed combination of user association policy, receive antenna, and interference mitigation technique. Based on previous findings, we therefore propose the combination of maximum-SINR based user association, directional antenna with fixed beamwidth, and ICIC with six coordinated BSs (e.g., $K=7$ ). We refer this combination of strategies as the interference-aware scheme. Meanwhile, the benchmark scheme is referred to as a combination of minimum-distance based user association, omni-directional antenna, and non-ICIC (i.e., $K=1$ ). As seen in Fig. 9, the coverage probability of AU improves from 0.335 to 0.964 ( $\sim$ threefold) at $-3.2815 \mathrm{~dB}$. Besides, if $K=7$, the TU's average rate improves from $110 \mathrm{Kbps}$ to $603 \mathrm{Kbps}$ 


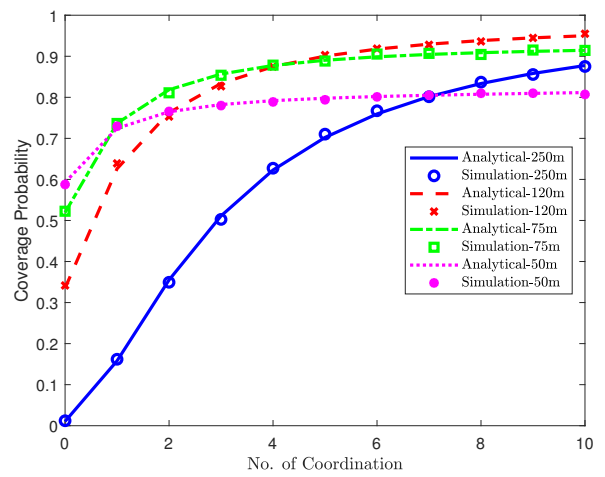

(a)

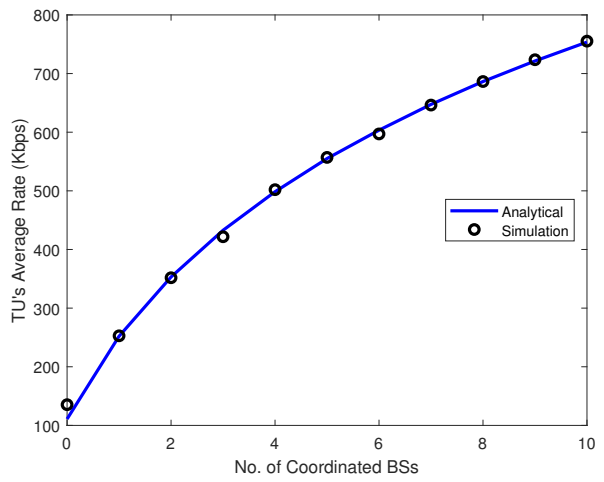

(b)

Figure 8: The impact of different number of coordinated BSs: a) coverage probabilities of the AU; b) TU's average rate.

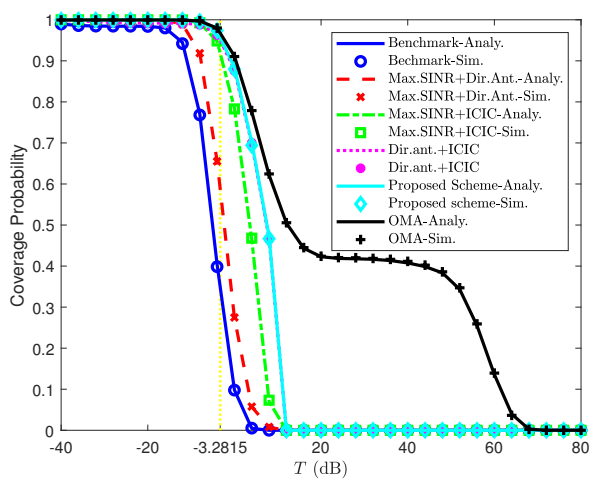

Figure 9: Coverage probabilities of the AU versus $T$ using different combinations of strategies.

( sixfold). One might also be curious about the performance of OMA. Thus, we consider the power coefficient $\rho_{u}=1$, where the entire resource block is used by AU only. In this case, the average rate of TU is zero. As seen, the AU achieves a substantial coverage probability for a very large $T$. This improvement is due to the combination of directional antenna and ICIC, which consequentially helps AU to experience zero ICI in some realizations. Nevertheless, the coverage probability of AU only increases by 0.009 (less than $0.1 \%$ ) at $-3.2815 \mathrm{~dB}$ as compared to the proposed scheme. Therefore, one may want to employ NOMA over OMA as it provides a similar level of reliability to AU but with a huge rate gain to TU.

\section{CONCLUSION}

In this paper, we studied the performance of downlink NOMA for cellular-connected UAVs. We presented a novel framework for the co-existence of AUs and TUs in cellular networks, where BSs are distributed using PPP. Furthermore, we considered two types of user association policies, two types of receive antennas, and a simple ICIC. The coverage probability and average rate of the $\mathrm{AU}$ and $\mathrm{TU}$ were derived. Using these expressions, we further analyzed the impact of different network parameters such as AU's altitude, TU's distance, power allocation, SIC constraints, user association policy, beamwidth, and number of coordinated BSs. Simulation and analytical results showed that an efficient usage of NOMA requires a combination of user association policy, receive antenna, and ICI mitigation technique. We therefore proposed a scheme that combines the use of maximumSINR based user association, directional antenna with fixed beamwidth, and ICIC. Our proposed scheme significantly improves the overall performance. Compared to the benchmark scheme, our proposed scheme improves the coverage probability of AU from 0.335 to 0.964 and TU's average rate from $110 \mathrm{Kbps}$ to $603 \mathrm{Kbps}$. Compared to OMA, our proposed scheme provides an additional rate of $603 \mathrm{Kbps}$ per $\mathrm{RB}$ to $\mathrm{TU}$, subject to a cost of degrading the AU's coverage probability by 0.009 at $-3.2815 \mathrm{~dB}$. Our findings suggest that NOMA is a promising solution for AUs and TUs for C\&C and data links, respectively, in future aerial-terrestrial networks.

\section{ApPendix A: Proof of Lemma 2}

For ease of expositions, let $D_{0}$ be the disk $\mathcal{B}\left(\hat{\boldsymbol{w}}^{\mathrm{AU}}, r_{u}\right)$ and $D_{1}$ be the disk $\mathcal{B}\left(\hat{\boldsymbol{w}}_{b}^{\mathrm{BS}}, r\right)$ see, Fig. 10. Conditioning on $r_{u}$, the CDF of $r$ is:

$$
\begin{aligned}
F_{r \mid r_{u}}\left(r_{1}\right) & =\mathcal{P}\left(r \leq r_{1} \mid r_{u}=r_{0}\right) \\
& =1-\exp \left(-\lambda \mathcal{A}\left(r_{1} \mid r_{0}\right)\right),
\end{aligned}
$$

where $\mathcal{A}\left(r_{1} \mid r_{0}\right)$ is the area of $D_{1}-\left(D_{0} \cap D_{1}\right)$. Using the law of cosines, the area of $\mathcal{A}\left(r_{1} \mid r_{0}\right)$ can be derived as follows:

$$
\mathcal{A}\left(r_{1} \mid r_{0}\right)= \begin{cases}k, & r_{1} \leq 2 r_{0}, \\ \pi\left(r_{1}^{2}-r_{0}^{2}\right), & r_{1}>2 r_{0},\end{cases}
$$

where $k=\pi r_{1}^{2}-\sum_{i=0}^{1}\left(r_{i}^{2} \cos ^{-1}\left(\frac{c_{i}}{r_{i}}\right)-c_{i} \sqrt{r_{i}^{2}-c_{i}^{2}}\right), c_{0}=$ $\frac{2 r_{0}^{2}-r_{1}^{2}}{2 r_{0}}$, and $c_{1}=\frac{r_{1}^{2}}{2 r_{0}}$.

By taking the derivative of (39) w.r.t. $r_{1}$, the conditional pdf of $r$ is obtained as:

$$
f_{r \mid r_{u}}\left(r_{1}\right)= \begin{cases}\lambda k_{1} \exp (-\lambda k), & r_{1} \leq 2 r_{0} \\ 2 \lambda \pi r_{1} \exp \left(-\lambda \pi\left(r_{1}^{2}-r_{0}^{2}\right)\right), & r_{1}>2 r_{0}\end{cases}
$$

where

$$
k_{1}=2 \pi r_{1}-2 r_{1} \cos ^{-1}\left(\frac{r_{1}}{2 r_{0}}\right) .
$$

By scaling $r \rightarrow \frac{\gamma r}{2}$, we obtain the conditional pdf of $r_{t}$ given $r_{u}$ as expressed in (29). 


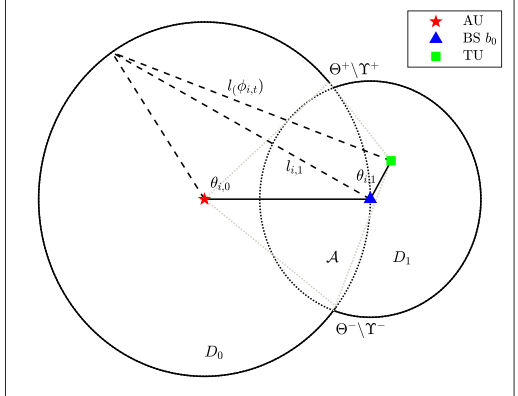

(i)

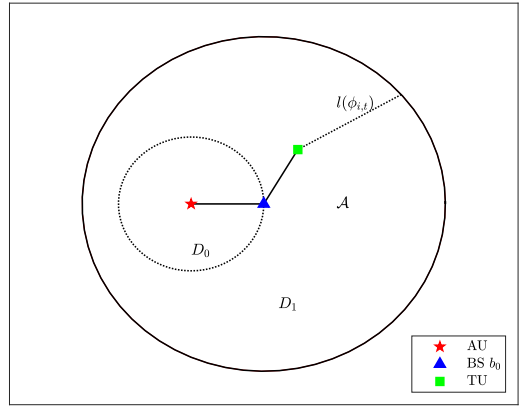

(ii)

Figure 10: Cases: (i) $r_{1} \leq 2 r_{0}$; (ii) $r_{1}>2 r_{0}$.

\section{APPENDIX B: PROOF OF THEOREM 1}

The coverage probability of the AU is defined as

$$
\mathcal{P}_{\text {cov }, u}(T)=\mathcal{P}\left(S I N R_{u}>T\right),
$$

where $S I N R_{u}$ is the SINR of the AU regardless of the link type, $v$, and $T$ is a SINR threshold. Conditioning on $r_{u}$ and $v$, the coverage probability of $\mathrm{AU}$ is defined as:

$$
\begin{aligned}
\mathcal{P}_{\text {cov }, u}(T)= & \int_{0}^{r_{\max }} \sum_{\forall v} \mathcal{P}\left(S I N R_{u}^{v}>T \mid r_{u}\right) \\
& \times P_{u}^{v} F_{p, r_{u}}^{v}\left(r_{\max }\right) \hat{f}_{r_{u}}^{v}\left(r_{0}\right) d r_{0},
\end{aligned}
$$

where $\mathcal{P}\left(S I N R_{u}^{v}>T \mid r_{u}\right)$ is the conditional coverage probability of AU for $v$ link. Substituting (11), the conditional probability of the AU for $v$ link is obtained as:

$$
\begin{aligned}
& \mathcal{P}\left(S I N R_{u}^{v}>T \mid r_{u}\right)
\end{aligned}
$$

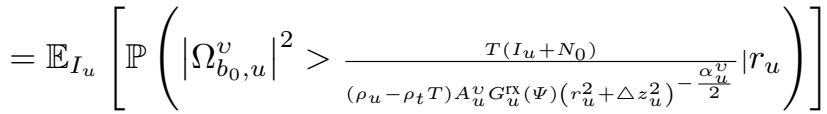

$$
\begin{aligned}
& \stackrel{(\mathrm{a})}{=} \mathbb{E}_{I_{u}}\left[\sum_{i=0}^{m_{u}^{v}-1} \frac{\left(-q_{v}\right)^{i}}{i !}\left(I_{u}+N_{0}\right)^{i} \exp \left(-q_{v}\left(I_{u}+N_{0}\right)\right) \mid r_{u}\right] \\
& \stackrel{(\mathrm{b})}{=} \sum_{i=0}^{m_{u}^{v}-1} s_{i}^{v} \mathbb{E}_{I_{u}}\left[I_{u}^{i} \exp \left(-q_{v} I_{u}\right) \mid r_{u}\right] \\
& \stackrel{\text { (c) }}{=} \sum_{i=0}^{m_{u}^{v}-1}(-1)^{i} s_{i}^{v} \frac{\partial^{i}}{\partial q_{v}^{i}} \mathcal{L}_{I_{u}}^{v}\left(q_{v}\right) \text {, }
\end{aligned}
$$

where $I_{u}=\sum_{b \in \Phi_{\mathcal{I}}^{u}} \chi_{b}\left|h_{b, u}^{v}\right|^{2}, s_{i}^{v}=\frac{\exp \left(-q_{v} N_{0}\right)}{i !} \sum_{j=i}^{m_{u}^{v}-1} \frac{N_{0}^{j-i} q_{v}^{i}}{(j-i) !}$, and $q_{v}=\frac{T}{\left(\rho_{u}-\rho_{t} T\right) A_{u}^{v} G_{u}^{\mathrm{rx}}(\Psi)\left(r_{u}^{2}+\Delta z_{u}^{2}\right)^{-\frac{\alpha_{u}^{v}}{2}} \theta_{u}^{v}}$. In (45), (a) is obtained using the ccdf of $\left|\Omega_{b_{0}, u}^{v}\right|^{2}$, (b) is obtained using binomial theorem, and (c) is obtained using the fact that the expectation term is equivalent to the $i$ th derivative of the Laplace transform of interference at AU.

The Laplace transform of interference at $\mathrm{AU}$ is:

$$
\begin{aligned}
& \mathcal{L}_{I_{u}}^{v}\left(q_{v}\right)=\mathbb{E}_{I_{u}}\left[\exp \left(-q_{v} I_{u}\right)\right] \\
& \stackrel{(\mathrm{a})}{=} \mathbb{E}_{\Phi_{I_{u}}^{\mathrm{L}}, \chi_{b}}\left[\prod_{b \in \Phi_{I_{u}}^{\mathrm{L}}} \mathbb{E}_{\Omega_{b, u}^{\mathrm{L}}}\left[\exp \left(-q_{v} \chi_{b} \Xi_{b, u}^{\mathrm{L}}\left|\Omega_{b, u}^{\mathrm{L}}\right|^{2}\right)\right]\right] \times \\
& \quad \mathbb{E}_{\Phi_{I_{u}}^{\mathrm{N}}, \chi_{b}}\left[\prod_{b \in \Phi_{I_{u}}^{\mathrm{N}}} \mathbb{E}_{\Omega_{b, u}^{\mathrm{N}}}\left[\exp \left(-q_{v} \chi_{b} \Xi_{b, u}^{\mathrm{N}}\left|\Omega_{b, u}^{\mathrm{N}}\right|^{2}\right)\right]\right] \\
& \stackrel{(\mathrm{b})}{=} \mathbb{E}_{\Phi_{I_{u}, \chi_{b}}^{\mathrm{L}}}\left[\prod_{b \in \Phi_{I_{u}}^{\mathrm{L}}} g^{\mathrm{L}}\left(q_{v}\right)^{\chi_{b}}\right] \mathbb{E}_{\Phi_{I_{u}}^{\mathrm{N}}, \chi_{b}}\left[\prod_{b \in \Phi_{I_{u}}^{\mathrm{N}}} g^{\mathrm{N}}\left(q_{v}\right)^{\chi_{b}}\right] \\
& \stackrel{(\mathrm{c})}{=} \exp \left(\sum_{\forall \tilde{v}} \int_{R^{v, \tilde{v}}\left(r_{u}\right)}^{r_{\max }}-2 \pi \frac{\lambda}{K} \times\right. \\
& \left.\mathcal{P}_{b, u}^{\tilde{v}}\left(r_{b, u}, \Delta z_{u}\right)\left(1-g^{\tilde{v}}\left(q_{v}\right)\right) r_{b, u} d r_{b, u}\right),
\end{aligned}
$$

where $g^{\tilde{v}}\left(q_{v}\right)=\left(1+q_{v} A_{u}^{\tilde{v}} G_{u}^{\mathrm{rx}}(\Psi)\left(r_{u}^{2}+\triangle z_{u}^{2}\right)^{-\frac{\alpha_{u}^{\tilde{v}}}{2}} \theta_{u}^{\tilde{v}}\right)^{-m_{u}^{\tilde{v}}}$, $\tilde{v} \in\{\mathrm{L}, \mathrm{N}\}$, and $\Phi_{I}^{v}$ is the set of interfering BSs that establishes $v$ links to the AU. Besides, $R^{v, \tilde{v}}\left(r_{u}\right)=r_{u}$ if $v=\tilde{v}$ and $R^{v, \tilde{v}}\left(r_{u}\right)=R^{v}\left(r_{0}\right)$ if $v \neq \tilde{v}$. In (46), (a) is obtained using thinning, (b) is obtained using the MGF of $\left|\Omega_{b, u}^{v}\right|^{2}$, and (c) is obtained using the PGFL.

\section{ApPendix C: PROOF OF LEMMA 3}

The $i^{\text {th }}$ derivative of $\mathcal{L}^{v} I_{u}\left(q_{v}\right)$ is:

$$
\begin{aligned}
& \frac{\partial^{i}}{\partial q_{v}^{i}} \mathcal{L}^{v}{ }_{I_{u}}\left(q_{v}\right) \\
& =\frac{\partial^{i}}{\partial q_{v}^{i}} \exp \left(\sum_{\forall \tilde{v}} \int_{R^{v, \tilde{v}}\left(r_{u}\right)}^{r_{\max }}-2 \pi \frac{\lambda}{K} \times\right. \\
& \left.\quad \mathcal{P}_{b, u}^{\tilde{v}}\left(r_{b, u}, \Delta z_{u}\right)\left(1-g^{\tilde{v}}\left(q_{v}\right)\right) r_{b, u} d r_{b, u}\right) \\
& \stackrel{(a)}{=} \sum_{j=1}^{i} \exp ^{(j)}\left(\mathcal{J}\left(q_{v}\right)\right) B_{i, j}\left(\mathcal{J}^{(1)}\left(q_{v}\right), \mathcal{J}^{(2)}\left(q_{v}\right), \ldots, \mathcal{J}^{(j)}\left(q_{v}\right)\right) \\
& \stackrel{(b)}{=} \exp \left(\mathcal{J}\left(q_{v}\right)\right) \sum_{j=1}^{i} B_{i, j}\left(\mathcal{J}^{(1)}\left(q_{v}\right), \mathcal{J}^{(2)}\left(q_{v}\right), \ldots, \mathcal{J}^{(j)}\left(q_{v}\right)\right) \\
& \stackrel{(c)}{=} \mathcal{L}^{v}{ }_{I_{u}}\left(q_{v}\right) B_{i}\left(\mathcal{J}^{(1)}\left(q_{v}\right), \mathcal{J}^{(2)}\left(q_{v}\right), \ldots, \mathcal{J}^{(i)}\left(q_{v}\right)\right),
\end{aligned}
$$

where

$$
\begin{aligned}
& \mathcal{J}\left(q_{v}\right)=\sum_{\forall \tilde{v}} \int_{R^{v}, \tilde{v}\left(r_{u}\right)}^{r_{\max }}-2 \pi \frac{\lambda}{K} \times \\
& \mathcal{P}_{b, u}^{\tilde{v}}\left(r_{b, u}, \Delta z_{u}\right)\left(1-g^{\tilde{v}}\left(q_{v}\right)\right) r_{b, u} d r_{b, u} .
\end{aligned}
$$

In (47), (a) is obtained using Faa' di Bruno formula [43], (b) is obtained using the fact that any $j^{\text {th }}$ derivative of an exponential 
function is itself, and (c) is obtained by the definition of a complete Bell polynomial. Furthermore, the $j^{\text {th }}$ derivative of $\mathcal{J}\left(q_{v}\right)$ w.r.t. $q_{v}$ is obtained as:

$$
\begin{aligned}
\mathcal{J}^{(j)}\left(q_{v}\right)= & \frac{\partial^{j}}{\partial q_{v}^{j}}\left[\sum_{\forall \tilde{v}} \int_{R^{v, \tilde{v}}\left(r_{u}\right)}^{r_{\max }}-2 \pi \frac{\lambda}{K} \times\right. \\
& \left.\mathcal{P}_{b, u}^{\tilde{v}}\left(r_{b, u}, \Delta z_{u}\right)\left(1-g^{\tilde{v}}\left(q_{v}\right)\right) r_{b, u} d r_{b, u}\right] \\
= & \sum_{\forall \tilde{v}}\left[\int_{R^{v, \tilde{v}}\left(r_{u}\right)}^{r_{\max }} \frac{\partial^{j}}{\partial q_{v}^{j}}-2 \pi \frac{\lambda}{K} \times\right. \\
& \left.\mathcal{P}_{b, u}^{\tilde{v}}\left(r_{b, u}, \Delta z_{u}\right)\left(1-g^{\tilde{v}}\left(q_{v}\right)\right) r_{b, u} d r_{b, u}\right],
\end{aligned}
$$

where the last line is obtained using the Leibniz's integral rule.

\section{APPEndiX D: Proof OF THEOREM 2}

Recall that the TU needs to remove the AU's message to decode its own message. Hence, the coverage probability of the TU is:

$$
\mathcal{P}_{\text {cov }, t}(T)=\mathcal{P}\left(S I N R_{t}^{(u)}>T_{\min }, S I N R_{t}>T\right),
$$

where $T_{\min }=2^{\frac{R_{\min }}{B_{\mathrm{w}}}}-1, R_{\min }$ and $B_{\mathrm{w}}$ are the targeted data rates required to successfully decode the AU's message, and the system bandwidth, respectively. Subject to $\theta_{t}, r_{t}, r_{u}$, the downlink coverage probability of TU being at a distance of $r_{t}$ away from BS $b_{0}$ over NOMA is:

$\mathcal{P}_{\text {cov }, t}(T)=$

$\int_{0}^{2 \pi} \int_{0}^{\infty} \int_{0}^{\infty} \mathcal{P}\left(S I N R_{t}^{(u)}>T_{\min }, S I N R_{t}>T \mid \theta_{t}, r_{t}, r_{u}\right)$ $\times \frac{1}{2 \pi} f_{r_{t} \mid r_{u}}\left(r_{2}\right) f_{r_{u}}\left(r_{0}\right) d r_{2} d r_{0} d \theta_{t}$

By substituting (12)-(13), the conditional coverage probability of TU is:

$$
\begin{aligned}
& \mathcal{P}\left(S I N R_{t}^{(u)}>T_{\min }, S I N R_{t}>T \mid \theta_{t}, r_{t}, r_{u}\right) \\
= & \mathcal{P}\left(\left|\Omega_{b_{0}, t}\right|^{2}>\frac{T_{\min }\left(I_{t}+N_{0}\right)}{\left(\rho_{u}-\rho_{t} T_{\min }\right) A_{t}\left(r_{t}^{2}+\Delta z_{t}^{2}\right)^{-\frac{\alpha}{2}}},\right. \\
& \left.\left|\Omega_{b_{0}, t}\right|^{2}>\frac{T\left(I_{t}+N_{0}\right)}{\rho_{t} A_{t}\left(r_{t}^{2}+\Delta z_{t}^{2}\right)^{-\frac{\alpha}{2}}} \mid \theta_{t}, r_{t}, r_{u}\right) \\
= & \mathcal{P}\left(\left|\Omega_{b_{0}, t}\right|^{2}>q_{t}\left(I_{t}+N_{0}\right) \mid \theta_{t}, r_{t}, r_{u}\right) \\
\stackrel{(\mathrm{a})}{=} & \exp \left(-q_{t} N_{0}\right) \mathbb{E}_{I_{t}}\left[\exp \left(-q_{t} I_{t}\right)\right] \\
= & \exp \left(-q_{t} N_{0}\right) \mathcal{L}_{I_{t}}\left(q_{t}\right),
\end{aligned}
$$

where $q_{t}=\max \left(\frac{T}{\rho_{t} A_{t}\left(r_{t}^{2}+\Delta z_{t}^{2}\right)^{-\frac{\alpha}{2}}}, \frac{T_{\min }}{\left(\rho_{u}-\rho_{t} T_{\min }\right) A_{t}\left(r_{t}^{2}+\triangle z_{t}^{2}\right)^{-\frac{\alpha}{2}}}\right)$, $\mathcal{L}_{I_{t}}\left(q_{t}\right)=\mathbb{E}_{I_{t}}\left[\exp \left(-q_{t} I_{t}\right)\right]$, and (a) is obtained using the ccdf of $\left|\Omega_{b_{0}, t}\right|^{2}$.

\section{REFERENCES}

[1] W. K. New, C. Y. Leow, K. Navaie, Y. Sun, and Z. Ding, "Downlink NOMA for coexistence of aerial and terrestrial users: Stochastic geometry analysis," in GLOBECOM 2020 - 2020 IEEE Global Communications Conference, pp. 1-6, 2020.

[2] X. Lin, V. Yajnanarayana, S. D. Muruganathan, S. Gao, H. Asplund, H. Maattanen, M. Bergstrom, S. Euler, and Y. E. Wang, "The sky is not the limit: LTE for unmanned aerial vehicles," IEEE Communications Magazine, vol. 56, pp. 204-210, Apr. 2018.

[3] Y. Zeng, Q. Wu, and R. Zhang, "Accessing from the sky: A tutorial on UAV communications for 5G and beyond," Proceedings of the IEEE, vol. 107, pp. 2327-2375, Dec 2019.

[4] "Study on enhanced LTE support for aerial vehicles," tech. rep., 3rd Generation Partnership Project, Dec. 2017.

[5] "LTE unmanned aircraft systems: Trial report v1.0.1," tech. rep., Qualcomm Technologies, Inc, May 2017.

[6] B. V. D. Bergh, A. Chiumento, and S. Pollin, "LTE in the sky: Trading off propagation benefits with interference costs for aerial nodes," IEEE Communications Magazine, vol. 54, pp. 44-50, May 2016.

[7] I. Kovacs, R. Amorim, H. C. Nguyen, J. Wigard, and P. Mogensen, "Interference analysis for UAV connectivity over LTE using aerial radio measurements," in Proc. 2017 IEEE 86th Vehicular Technology Conference (VTC-Fall), pp. 1-6, Sept 2017.

[8] M. M. Azari, F. Rosas, A. Chiumento, and S. Pollin, "Coexistence of terrestrial and aerial users in cellular networks," in 2017 IEEE Globecom Workshops (GC Wkshps), pp. 1-6, Dec 2017.

[9] M. M. Azari, F. Rosas, and S. Pollin, "Reshaping cellular networks for the sky: Major factors and feasibility," in 2018 IEEE International Conference on Communications (ICC), pp. 1-7, May 2018.

[10] V. Yajnanarayana, Y. Eric Wang, S. Gao, S. Muruganathan, and X. Lin Ericsson, "Interference mitigation methods for unmanned aerial vehicles served by cellular networks," in 2018 IEEE $5 G$ World Forum (5GWF), pp. 118-122, July 2018.

[11] S. Euler, H. Maattanen, X. Lin, Z. Zou, M. Bergstrom, and J. Sedin, "Mobility support for cellular connected unmanned aerial vehicles: Performance and analysis," in 2019 IEEE Wireless Communications and Networking Conference (WCNC), pp. 1-6, April 2019.

[12] W. Mei and R. Zhang, "Cooperative downlink interference transmission and cancellation for cellular-connected UAV: A divide-and-conquer approach," IEEE Transactions on Communications, vol. 68, no. 2, pp. 1297-1311, 2020.

[13] M. M. Azari, F. Rosas, and S. Pollin, "Cellular connectivity for UAVs: Network modeling, performance analysis, and design guidelines," IEEE Transactions on Wireless Communications, vol. 18, pp. 3366-3381, July 2019.

[14] Z. Chen, Z. Ding, X. Dai, and R. Zhang, "An optimization perspective of the superiority of NOMA compared to conventional OMA," IEEE Transactions on Signal Processing, vol. 65, pp. 5191-5202, Oct 2017.

[15] Y. Liu, G. Pan, H. Zhang, and M. Song, "On the capacity comparison between MIMO-NOMA and MIMO-OMA," IEEE Access, vol. 4 pp. 2123-2129, 2016.

[16] W. Mei and R. Zhang, "Uplink cooperative NOMA for cellularconnected UAV," IEEE Journal of Selected Topics in Signal Processing, pp. 1-1, 2019.

[17] L. Liu, S. Zhang, and R. Zhang, "Exploiting NOMA for multi-beam UAV communication in cellular uplink," in ICC 2019 - 2019 IEEE International Conference on Communications (ICC), pp. 1-6, May 2019.

[18] X. Mu, Y. Liu, L. Guo, and J. Lin, "Non-orthogonal multiple access for air-to-ground communication," IEEE Transactions on Communications, pp. $1-1,2020$

[19] N. Senadhira, S. Durrani, X. Zhou, N. Yang, and M. Ding, "Uplink NOMA for cellular-connected UAV: Impact of UAV trajectories and altitude," IEEE Transactions on Communications, pp. 1-1, 2020.

[20] T. Hou, Y. Liu, Z. Song, X. Sun, and Y. Chen, "NOMA-enhanced terrestrial and aerial IoT networks with partial CSI," IEEE Internet of Things Journal, vol. 7, no. 4, pp. 3254-3266, 2020.

[21] W. K. New, C. Y. Leow, K. Navaie, and Z. Ding, "Robust nonorthogonal multiple access for aerial and ground users," IEEE Transactions on Wireless Communications, vol. 19, no. 7, pp. 4793-4805, 2020

[22] S. K. Zaidi, S. F. Hasan, and X. Gui, "Outage analysis of groundaerial NOMA with distinct instantaneous channel gain ranking," IEEE Transactions on Vehicular Technology, vol. 68, pp. 10775-10790, Nov 2019. 
[23] W. Mei and R. Zhang, "Cooperative NOMA for downlink asymmetric interference cancellation," IEEE Wireless Communications Letters, 2020.

[24] W. K. New, C. Y. Leow, K. Navaie, Y. Sun, and Z. Ding, "Application of NOMA for cellular-connected UAVs: Opportunities and challenges," Information Sciences, vol. 64, no. 140302, pp. 1-140302, 2021.

[25] M. Haenggi, Stochastic Geometry for Wireless Networks. Cambridge University Press, 2012.

[26] J. G. Andrews, A. K. Gupta, and H. S. Dhillon, "A primer on cellular network analysis using stochastic geometry," arXiv preprint arXiv:1604.03183, 2016.

[27] F. Baccelli and B. Błaszczyszyn, Stochastic Geometry and Wireless Networks, vol. 1. Now Publishers Inc, 2009.

[28] M. Haenggi and R. K. Ganti, Interference in Large Wireless Networks. Now Publishers Inc, 2009.

[29] H. ElSawy, A. Sultan-Salem, M. Alouini, and M. Z. Win, "Modeling and analysis of cellular networks using stochastic geometry: A tutorial," IEEE Communications Surveys Tutorials, vol. 19, no. 1, pp. 167-203, 2017.

[30] M. Salehi and E. Hossain, "On coverage probability in uplink NOMA with instantaneous signal power-based user ranking," IEEE Wireless Communications Letters, vol. 8, no. 6, pp. 1683-1687, 2019.

[31] S. Zhang, X. Xu, H. Wang, J. Peng, D. Zhang, and K. Huang, "Enhancing the physical layer security of uplink non-orthogonal multiple access in cellular Internet of Things," IEEE Access, vol. 6, pp. 58405-58417, 2018.

[32] M. Salehi, H. Tabassum, and E. Hossain, "Meta distribution of SIR in large-scale uplink and downlink NOMA networks," IEEE Transactions on Communications, vol. 67, no. 4, pp. 3009-3025, 2019.

[33] Y. Sun, Z. Ding, X. Dai, K. Navaie, and D. K. C. So, "Performance of downlink NOMA in vehicular communication networks: An analysis based on Poisson Line Cox point process," IEEE Transactions on Vehicular Technology, vol. 69, no. 11, pp. 14001-14006, 2020.

[34] Y. Sun, Z. Ding, and X. Dai, "On the performance of downlink NOMA in multi-cell mmWave networks," IEEE Communications Letters, vol. 22, no. 11, pp. 2366-2369, 2018.

[35] C. A. Balanis, Antenna Theory: Analysis and Design, 3rd Edition. John wiley \& sons, 2005.

[36] H. He, S. Zhang, Y. Zeng, and R. Zhang, "Joint altitude and beamwidth optimization for UAV-enabled multiuser communications," IEEE Communications Letters, vol. 22, pp. 344-347, Feb 2018.

[37] N. Cherif, M. Alzenad, H. Yanikomeroglu, and A. Yongacoglu, "Downlink coverage and rate analysis of an aerial user in integrated aerial and terrestrial networks," arXiv preprint arXiv:1905.11934, 2019.

[38] J. G. Andrews, F. Baccelli, and R. K. Ganti, "A tractable approach to coverage and rate in cellular networks," IEEE Transactions on Communications, vol. 59, no. 11, pp. 3122-3134, 2011.

[39] A. Mahmud and K. A. Hamdi, "A unified framework for the analysis of fractional frequency reuse techniques," IEEE Transactions on Communications, vol. 62, no. 10, pp. 3692-3705, 2014.

[40] X. Zhang and M. Haenggi, "A stochastic geometry analysis of inter-cell interference coordination and intra-cell diversity," IEEE Transactions on Wireless Communications, vol. 13, no. 12, pp. 6655-6669, 2014.

[41] J. Yoon and G. Hwang, "Distance-based inter-cell interference coordination in small cell networks: Stochastic geometry modeling and analysis," IEEE Transactions on Wireless Communications, vol. 17, no. 6, pp. 4089-4103, 2018.

[42] T. Bai and R. W. Heath, "Coverage and rate analysis for millimeter-wave cellular networks," IEEE Transactions on Wireless Communications, vol. 14, no. 2, pp. 1100-1114, 2015.

[43] T. Kim, D. S. Kim, and G.-W. Jang, "On central complete and incomplete Bell polynomials I," Symmetry, vol. 11, no. 2, p. 288, 2019.

[44] F. Yilmaz and M. Alouini, "A unified MGF-based capacity analysis of diversity combiners over generalized fading channels," IEEE Transactions on Communications, vol. 60, no. 3, pp. 862-875, 2012.

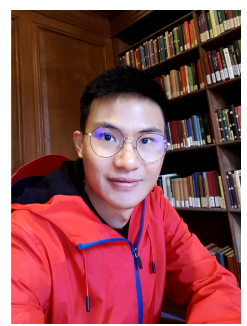

Wee Kiat New received his B.IT in Data Communications and Networking from Multimedia University and M.Eng.Sc from University Malaya in 2012 and 2015, respectively. He is currently pursuing his $\mathrm{Ph} . \mathrm{D}$ in Electrical Engineering at Wireless Communication Centre, Universiti Teknologi Malaysia. He was a visiting researcher at School of Computing and Communications, Lancaster University, and KIOS Research and Innovation Centre, University of Cyprus. His current research interests are optimization, stochastic processes, non-orthogonal multiple access (NOMA), and unmanned aerial vehicle (UAV) communication.
Chee Yen (Bruce) Leow obtained the B.Eng. degree in Computer Engineering from Universiti Teknologi Malaysia (UTM) and the Ph.D. degree from Imperial College London in 2007 and 2011, respectively. Since July 2007, he has been an academic staff with the School of Electrical Engineering, Faculty of Engineering, UTM. He is currently an Associate Professor in the School and a Research Fellow in the Wireless Communication Centre UTM and UTM-Ericsson Innovation Centre for 5G. His research interests include non-orthogonal multiple access, UAV communication, MIMO, hybrid beamforming, physical layer security, wireless power transfer and prototype development using software defined radio, for $5 \mathrm{G}$ and IoT applications.

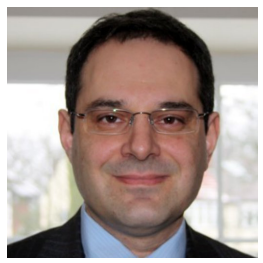

Keivan Navaie is with the School of Computing and Communications, Lancaster University, UK. His research seeks innovative inter-disciplinary solutions to address fundamental technical and design challenges in Intelligent Cyber Physical Systems and their integrations into day-to-day life to improve its sustainability and anti-fragility. Keivan's research covers topics across the following disciplines: Distributed Cloud and Edge Computing, Connectivity Resilience in Cyber-Physical Systems and IoT, Applications of Machine Learning and Artificial Intelligence, and Cognitive Communications Networks.

Keivan is on the editorial board of the IEEE Comm. Letters, IEEE Comm. Surveys and Tutorials, Trans. on Wireless Comm., and IEEE Access. He is also an advocate of evidence-based policy making through his involvement with the EU Environment Agency, and EU Parliament as an Independent Scientific Expert. Dr. Navaie is a Senior Member of the IEEE, and a Chartered Engineer in the UK.

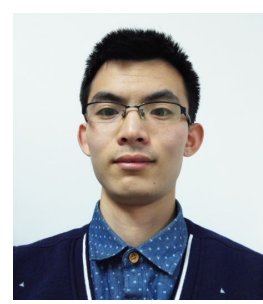

Yanshi Sun received the B.Eng. degree from the University of Science and Technology of China in 2016. He is currently working toward the Ph.D. degree in communication and information systems at the School of Information Science and Technology, University of Science and Technology of China. His current research interests include stochastic geometry, non-orthogonal multiple access (NOMA) systems, and unmanned aerial vehicles (UAVs).

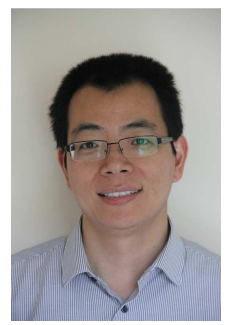

Zhiguo Ding (S'03-M'05-F20) received his B.Eng in Electrical Engineering from the Beijing University of Posts and Telecommunications in 2000, and the Ph.D degree in Electrical Engineering from Imperial College London in 2005. From Jul. 2005 to Apr. 2018, he was working in Queen's University Belfast, Imperial College, Newcastle University and Lancaster University. Since Apr. 2018, he has been with the University of Manchester as a Professor in Communications. From Oct. 2012 to Sept. 2021, he has also been an academic visitor in Princeton University. Dr Ding' research interests are 5G networks, game theory, cooperative and energy harvesting networks and statistical signal processing.

$\mathrm{He}$ is serving as an Area Editor for the IEEE Open Journal of the Communications Society, an Editor for IEEE Transactions on Vehicular Technology, and Journal of Wireless Communications and Mobile Computing, and was an Editor for IEEE Wireless Communication Letters, IEEE Transactions on Communications, IEEE Communication Letters from 2013 to 2016 . He recently received the EU Marie Curie Fellowship 2012-2014, the Top IEEE TVT Editor 2017, IEEE Heinrich Hertz Award 2018, IEEE Jack Neubauer Memorial Award 2018, IEEE Best Signal Processing Letter Award 2018 , and Friedrich Wilhelm Bessel Research Award 2020. He is a Fellow of the IEEE, a Distinguished Lecturer of IEEE ComSoc, and a Web of Science Highly Cited Researcher in two categories 2020. 